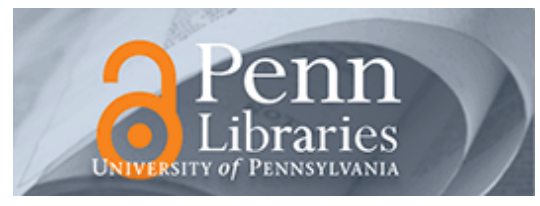

University of Pennsylvania

ScholarlyCommons

\title{
When Did We Become Human? Evolutionary Perspectives on the Emergence of the Modern Human Mind, Brain, and Culture
}

Theodore G. Schurr

University of Pennsylvania, tgschurr@sas.upenn.edu

Follow this and additional works at: https://repository.upenn.edu/anthro_papers

Part of the Anthropology Commons, and the Genetics Commons

\section{Recommended Citation (OVERRIDE)}

Schurr, T. G (2007). When Did We Become Human? Evolutionary Perspectives on the Emergence of the Modern Human Mind, Brain, and Culture. In G. Hatfield \& H. Pittman (Eds.), Evolution of Mind, Brain, and Culture (45-89). Philadelphia: University of Pennsylvania Museum Press.

This paper is posted at ScholarlyCommons. https://repository.upenn.edu/anthro_papers/165

For more information, please contact repository@pobox.upenn.edu. 


\section{When Did We Become Human? Evolutionary Perspectives on the Emergence of the Modern Human Mind, Brain, and Culture}

\section{Disciplines}

Anthropology | Genetics | Social and Behavioral Sciences 


\title{
When Did We Become Human? Evolutionary Perspectives on the Emergence of the Modern Human Mind, Brain, and Culture
}

\author{
THEODORE G. SCHURR
}

$\mathrm{O}$ ne of the most longstanding debates in the field of biological anthropology is when members of our lineage became "human." There is keen interest in knowing when we evolved the characteristics seen in our species, and which of these features truly makes us distinctive from other primates and especially earlier forms of hominins. Language, culture, tool use, brain size, and bipedalism have all been cited as traits that differentiate modern humans from other primate species. While it was once thought that these traits were uniquely human, we now understand most of them to be elaborations of similar features in other species, although with some specific manifestations for modern humans.

Counterexamples to modern human uniqueness seem to proliferate every day. For instance, chimps and orangs are now viewed not only as having "culture" (Whiten et al. 1999, Whiten, Horner, and Marshall-Pescini 2003, Hohmann and Fruth 2003, van Schaik et al. 2003, Whiten 2005, Lycett, Collard, and McGrew 2007, Horner et al. 2010), but, in the case of chimps, also an extensive cultural repertoire (Boesch, Hohman, and Marchant 2002, Boesch 2003) (Fig. 2.1). Field researchers have noted cultural patterns in chimps in 30 different behaviors, including anting, greeting gestures, and reconciliation (Whiten, Horner, and Marshall-Pescini 2003, Whiten 2005, Schöning et al. 2008). However, as S.E. Perry (2006:171) has noted, the evolution of culture in these species cannot be fully explained without determining "(a) how socioecological variables affect 


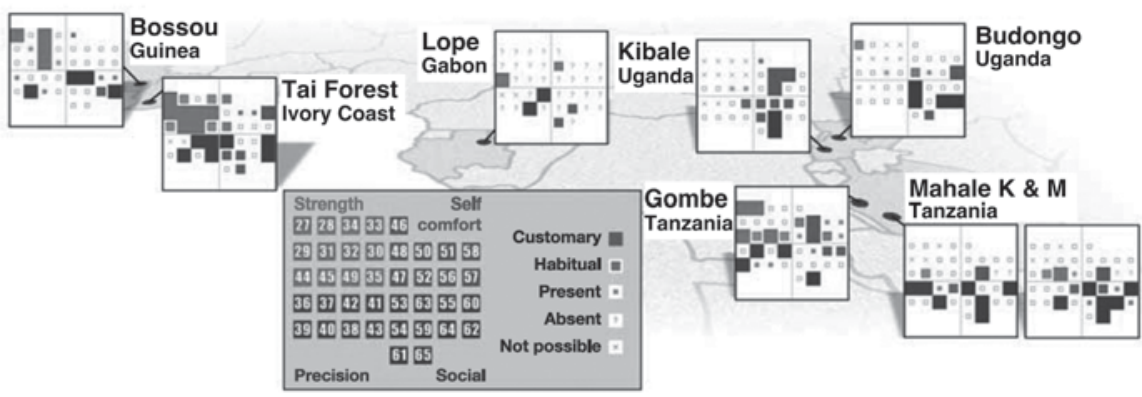

2.1. The cultures of wild chimpanzees. Each chimpanzee community has its own unique array of traditions that together constitute the local "culture." "Customary" acts are those typical in the community, "habitual" ones are less common but consistent with social transmission, and "absent" acts are those missing with no apparent straightforward environmental explanation. Traditions are defined as behavior patterns that are customary or habitual in at least one site but absent elsewhere. Transmission is attributed to social learning on the basis of a complex of circumstantial evidence. The numbers in cells refer to behavior patterns catalogued in Whiten et al. (2001:1489-1525). (Reprinted by permission from Macmillan Publishers Ltd: Nature 437 [2005], A. Whiten, pp. 52-55, Fig. 1.)

cultural transmission dynamics, (b) the proximate mechanisms by which social learning is achieved, (c) developmental studies of the role of social influence in acquiring behavioral traits, and (d) the fitness consequences of engaging in social learning."

Through numerous experimental studies, it is also clear that apes have incipient language abilities (Patterson and Linden 1981, Savage-Rumbaugh, Rumbaugh, and McDonald 1985, Savage-Rumbaugh et al. 1986, Miles 1990). Most apes have the capacity to comprehend and manipulate symbols, with some understanding of syntax. At the same time, apes cannot produce articulate speech like humans, and have a rudimentary understanding of grammar, although they are clearly able to communicate various emotions through vocalizations (Premack 2004, P. Lieberman 2007). Others suggest that clues to the emergence of human language may be found in the manual gestures, facial expressions, and vocal signals of chimps and bonobos (Pollick and de Waal 2007) or gorillas (Forrester 2008). Yet, it is still not clear whether apes understand anything about the unobservable mental states of other individuals (Penn and Povinelli 2007) or have shared intentionality as seen in humans (Tomasello et al. 2005b). 
Aside from cognitive abilities, there has also been a concerted effort to link social behavior and brain evolution. A number of studies attempted to associate the expansion of the neocortex to the social group complexity for different species (Humphrey 1976, Byrne and Whiten 1988, Dunbar 1992, 1993, 1995, 1998; but cf. L. Barrett, Henzi, and Rendall 2007). According to this social brain hypothesis, in a population containing multiple members of different sexes and ages, individuals need to understand the social relationships in the group, including both kin and non-kin (Byrne and Whiten 1988, Byrne 1996, Byrne and Bates 2007). Selection favored those individuals who were able to keep track of this complex set of relationships and remember information about others' demeanor, rank, kinship, and past history of giveand-take, while using this information in social problem-solving. Since it is the location where such higher cognitive functions take place, neocortex size and complexity would have been selected for under these conditions.

In addition, there is the issue of tool use by different primates, and the implications of deliberate manufacturing or employment of tools for the evolution of the brain. Indeed, thanks to many years of field research with wild populations of apes and monkeys, we now know that tool use occurs in most ape species (Fox, Sitompul, and van Schaik 1999, Hohmann and Fruth 2003, van Schaik et al. 2003, Breuer, Ndoundou-Hockemba, and Fishlock 2005, Mulcahy, Call, and Dunbar 2005, Hernandez-Aguilar, Moore, and Pickering 2007). There is more extensive use of tools for various purposes by chimps than by other great apes (e.g., Sanz, Morgan, and Gulick 2004, Sanz and Morgan 2007), which is consistent with chimps being our closest hominoid cousins. In fact, it appears that at least certain chimpanzees were capable of producing stone tools (Mercader et al. 2007). However, researchers have also observed tool use in certain New World monkey species, indicating that these behaviors are not confined to the Family Hominidae (e.g., Visalberghi et al. 2007). It further appears that tool use can be "taught" or transmitted between individuals, although not in as clearly a deliberate manner as with human teaching (Premack 2004).

All of this new evidence would seem to diminish the assertion of modern human uniqueness, despite clear quantitative differences between Homo sapiens and other primate species. In light of the changing perspective on human nature, this chapter provides an evolutionary context for assessing the evolution of mind, brain, and culture in modern humans, which is addressed in various ways in the chapters of this volume. It presents a view 
of human and primate evolution that reflects our current understanding of bio-behavioral variation in these species. Yet, even as these words are being written, this view is shifting very quickly with new information from ethology, neurobiology, genetics, and paleoanthropology. Even so, it is possible to highlight the major transitions in the evolution of primate and hominid species and explore their implications for the emergence of modern human behavior and cognitive capacities.

\section{HOMININ PHYLOGENY}

There have been many attempts to construct a phylogeny of fossil humans in an effort to determine the place of modern humans in nature. These trees have continued to change as new fossils are discovered and old fossils are reanalyzed, and the absolute dating of these fossils provides new insights into the chronological context of speciation events. One could rightly state that recent paleoanthropological research has been remarkably successful in providing new information about human ancestry and evolution and in filling some of the gaps in the hominin phylogeny that had persisted for some time.

The general outline of the evolution of hominin species ${ }^{1}$ is largely agreed upon. As illustrated in Figure 2.2, there are relatively few straight lines between the identified species, and more of a gradation between the different forms through time. We observe some correlation between brain size, tooth size, and bipedalism for all of these species, and that brain size increases while tooth size diminishes in the modern human lineage (genus Homo) over the past several million years.

In addition to discussions of the shape and complexity of hominin phylogeny, there is great interest in defining the starting point for this tree. Recent fossil discoveries have pushed back the origin of the hominin clade ${ }^{2}$ into the late Miocene, to 6-7 million years ago (mya). The oldest known potential hominin fossils have been attributed to Sahelanthropus tchadensis and come from Toros-Menalla in Chad (Brunet et al. 2002, 2005). This finding was unexpected because of the fact that so few hominin fossils had previously been discovered there. Some researchers assert that Sahelanthropus belongs to the stock that gave rise to both chimps and humans and is not the beginning point for the human lineage (e.g., Wolpoff et al. 2002).

The next-oldest species, Orrorin tugenensis, has been dated to 6.0 mya 


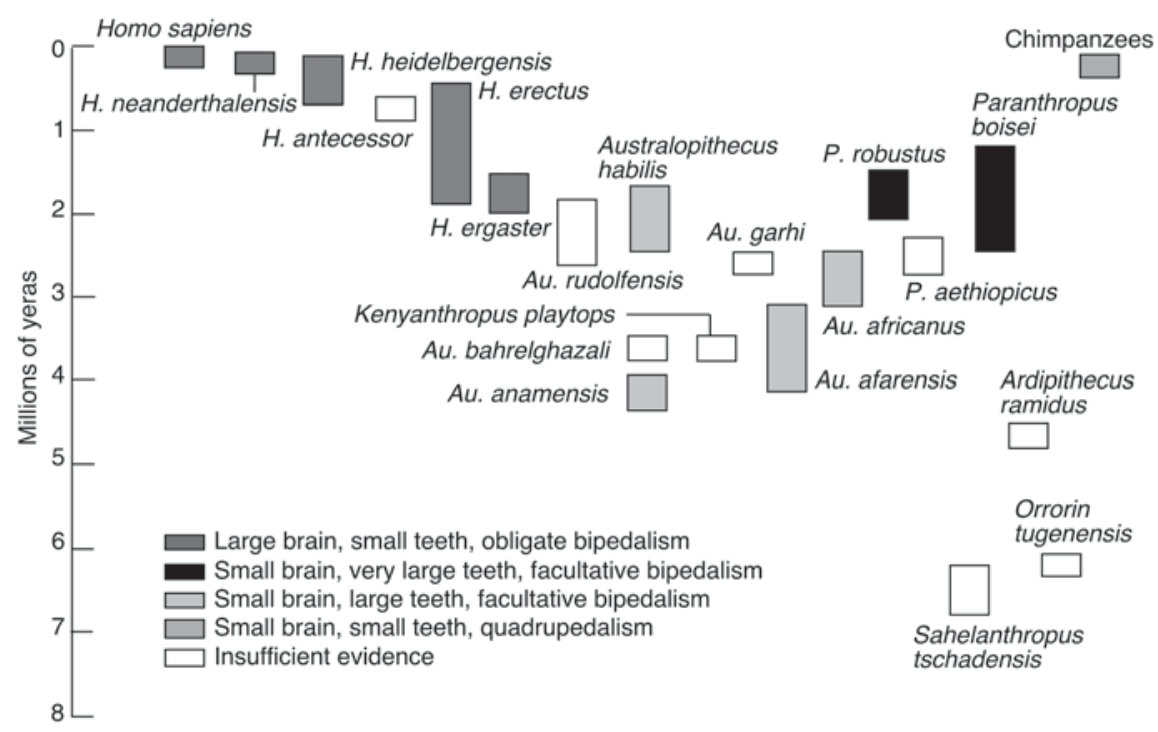

2.2. A current hominid phylogeny based on fossil evidence. Modern humans are shown at top left and the chimpanzee at top right. Extinct species are indicated with the dates of the earliest and latest fossil evidence; these are likely to change with the accumulation of more fossil evidence. Species are assigned to one of four categories, based on brain and cheek-tooth size, and inferred posture and locomotion. A fifth category, "insufficient evidence," indicates species that cannot be readily classified based on these features. (Modified after B. Wood, Nature 418 [2002]:133-35, Fig. 2; used with permission.)

(Senut et al. 2001, Pickford et al. 2002). As will be discussed below, this species shows clear postcranial evidence for bipedalism in the shape of the proximal femur, an adaptation seen as distinctive in hominins. This observation is quite important because it establishes the fact that upright posture and locomotion occurred very early in the evolution of the hominin lineage. There is debate over whether this "Millennium Man"- as well as Sahelanthropus - belongs to the human or human-chimp lineage (Haile-Selassie 2001).

Shortly after the appearance of Orrorin the earliest forms of Ardipithecus arose between 5.2-5.7 mya (Haile-Selassie, Suwa, and White 2004, Semaw et al. 2005). This species persisted until around 4.0 mya before giving way to australopithecines. The exact taxonomic status of this hominin is vigorously debated. These questions have arisen in part due to the fragmentary 
nature of the fossil evidence, and because this species seems to have both primitive and advanced features relative to later hominins and chimpanzees (Haile-Selassie, Suwa, and White 2004, Semaw et al. 2005).

Various forms of australopithecines emerged around 4.4 mya and persisted until some 1.0 mya (White et al. 2000, 2006). These species included both the more gracile forms of the genus Australopithecus and more robust forms in the genus Paranthropus. ${ }^{3}$ There is no clear consensus on the species that gave rise to Homo. Some favor A. garhi because of its possible association with Oldowan stone tools (Asfaw et al. 1999, de Heinzelin et al. 1999), although this view is not uniformly accepted. Others have suggested that a separate species (and genus), Kenyapithecus playtops, could be the precursor to the genus Homo (Leakey et al. 2001).

Regardless of its ancestor, the earliest forms of Homo appear to have evolved from an australopithecine stock around 1.8-2.3 mya (Gabunia et al. 2001, Vekua et al. 2002, Antón and Swisher 2004, Lordkipanidze et al. 2007). This transition is marked by a number of changes, such as the appearance of stone tools, an increase in brain size beyond the chimpanzee and australopithecine range, and other skeletal and behavioral attributes (McHenry and Coffing 2000). Meanwhile, the chimpanzee lineage continued to evolve and diverge, producing a number of subspecies over the course of the next several million years.

There is still considerable debate about the exact phylogenetic relationships among the early forms of Homo. This uncertainty arises in part because of fossils that were recently found in Dmanisi, Georgia. Attributed to Homo erectus, these fossils date to 1.8 mya and appear to have characteristics similar to both $H$. habilis and H. erectus (Gabunia et al. 2001, Vekua et al. 2002, Lordkipanidze et al. 2007). Some investigators have suggested that variation in early Homo may be partly ecogeographic in nature- that is, the Dmanisi specimens are simply smaller than those found in Africa-or that early African $H$. erectus fossils represent different populations of a single, highly variable species (Ruff 2002, D. Lieberman 2007). Alternatively, the Dmanisi fossils may represent a different species altogether (e.g., Vekua et al. 2002). In either case, early Homo probably had substantial levels of sexual dimorphism, ${ }^{4}$ as also seen in members of the genus Australopithecus (D. Lieberman 2007).

Given the temporal overlap in the appearance of $H$. habilis and H. erectus and their seemingly similar cognitive capabilities, as indicated by the use 
of simple stone tools by both taxa, there is some uncertainty about which hominin represents the precursor to $H$. erectus. For some time, $H$. habilis had been considered to be ancestral to $H$. erectus due to its having a vertical face, teeth of intermediate size between those of Australopithecus and H. erectus, and an intermediate-sized brain. One problem with this interpretation was that, until recently, the oldest fossils definitively attributed to $H$. habilis were only 1.9 million years old (Leakey, Tobias, and Napier 1964), a date which was not much earlier than the appearance of the oldest $H$. erectus fossils.

The discovery of a new H. habilis upper jaw dated to 1.44 mya (Spoor et al. 2007) further complicates the picture. The comparison of this specimen with other examples of early Homo demonstrates the distinctiveness of $H$. habilis and $H$. erectus regardless of their relative cranial size. This evidence plus the dates of the new fossils suggests that these taxa lived sympatrically ${ }^{5}$ in the same area for nearly 500,000 years (Spoor et al. 2007). However, it remains possible that $H$. habilis evolved before 2 mya, as a 2.33 mya upper jaw (AL 666) from Hadar, East Africa, is thought to belong to this species (Kimbel, Johanson, and Rak 1997).

Homo erectus represents a major transition in the genus Homo (Wood and Collard 1999, McHenry and Coffing 2000). This species took on modern human stature and limb proportions, became habitually bipedal, and began the ontogenetic ${ }^{6}$ shift that led to modern human life history (Leigh 1996, Bogin 2001, Aiello and Wells 2002). It has sometimes been subdivided into H. ergaster in Africa (ancestral form) and H. erectus in East Asia (derived form) to reflect subtle morphological and apparent behavioral differences between the specimens across the species' range, although not all investigators make this distinction (Antón and Swisher 2004). In addition, this species appears to have undergone major physiological changes to allow for greater mobility, changing dietary strategies, and new reproductive demands (Aiello and Wells 2002, Antón and Swisher 2004).

Current studies have also raised questions about the degree of behavioral and morphological modernity exhibited by early $H$. erectus. The species had small teeth, suggesting a more human-like diet, and relatively long legs and other features suited to walking and running (Wood and Collard 1999, McHenry and Coffing 2000, Pearson 2000, Ruff 2002, Bramble and Lieberman 2004), although these traits may also have been present in the Georgian hominins (Lordkipanidze et al. 2007). However, it appears that they matured more rapidly than modern humans do (Antón 2003), al- 
though some disagree with this interpretation (e.g., Clegg and Aiello 1999), and large increases in brain size occurred well after the species originated (Antón 2003, Antón and Swisher 2004, Schoenemann 2006). It is also likely that $H$. erectus was more sexually dimorphic than modern humans (Antón 2003, D. Lieberman 2007). These observations have important implications for reconstructing the bioenergetics, reproductive ecology, and social organization of this species.

During the later phases of $H$. erectus, the ancestral stock for later $H$. sapiens emerged. Called $H$. heidelbergensis by some, $H$. rhodesiensis by others (Hublin 2009), these archaic humans appeared by 600,000 years before present (ybp) in Africa, and then spread to other parts of the Old World. H. heidelbergensis in Africa continued to evolve in Africa, and eventually gave rise to anatomically modern humans (AMH), H. sapiens (Rightmire 1998, 2004, Stringer 2002a,b). According to Rightmire (2004), the changes in absolute and relative brain size in hominins from the Middle Pleistocene point to a speciation event in which $H$. erectus produced a daughter lineage, represented by $H$. heidelbergensis. Archaeological evidence from this period also indicates that these species differed in their technology and behavior from earlier hominins (Thieme 1997, Klein 2000b).

There is still some debate as to whether earlier archaic humans in Europe are related to $H$. heidelbergensis or instead represent a separate and earlier species of hominin, called $H$. antecessor (Bermúdez de Castro et al. 2004, Carbonell et al. 2008). This hominin form shows some differences from $H$. erectus, although being more archaic in some of its features from later $H$. heidelbergensis and Neandertals. Even if this species entered Europe some 800,000 years ago, it is not certain that this hominin eventually gave rise to the ancestral stock of Neandertals (Rightmire 2004, Bermúdez de Castro et al. 2004). "Classical" Neandertals lived in Europe and West Asia from $\sim 200,000-28,000$ ybp before disappearing, probably due to challenges presented by climate change, demography, and pressure from expanding AMH (Gravina, Mellars, and Ramsey 2005, Finlayson et al. 2006, Kuhn and Stiner 2006, Tzedakis et al. 2007).

There has been tremendous debate over the phylogenetic status of $\mathrm{Ne}$ andertals relative to AMH. The range of opinions includes Neandertals being the same species as AMH (Trinkhaus 1983, Wolpoff et al. 2001) or a separate species entirely (Tattersall and Schwartz 1999, Stringer 2002a,b). Most of the recent genetic data available from Neandertals and AMH- 
mitochondrial DNA, Y-chromosome, and autosomal DNA-indicate that there is considerable distance between these two hominins, perhaps at the species level (Serre et al. 2004, Caramelli et al. 2003, Green et al. 2006, Krause et al. 2007a).

The examination of craniofacial traits in these hominins also supports the view that Neandertals were a species distinct from early modern humans (Harvati, Frost, and McNulty 2004, Weaver, Roseman, and Stringer 2008), and had different developmental trajectories with taxon-specific patterns of ontogeny (Ponce de León and Zollikofer 2001, Ramirez Rossi and Bermúdez de Castro 2004). Collectively, these data imply that Neandertals were not the direct ancestors of any extant human populations, even if limited interbreeding were to have occurred between them (Duarte et al. 1999, Zilhão 2006).

However, more recent studies now suggest a somewhat different picture of the biological relationship between Neandertals and modern humans. Thanks to the technological advances of next generation sequencing, researchers have obtained a draft sequence of the Neandertal genome and compared it to those of modern humans and anthropoid primates to better define the genetic relationships between them. This research suggests that modern human and Neandertal DNA sequences diverged between 500,000 and 706,000 years ago based on genomic sequence data (Green et al. 2006, 2010, Noonan et al. 2006), and between $660,000 \pm 140,000$ years based on whole mtDNA genome sequence data (Green et al. 2008). However, the human and Neandertal ancestral populations appear to have split between 270,000 and 444,000 years ago (Noonan et al. 2006, Green et al. 2010), well before the emergence of modern humans in Africa.

Furthermore, the genomic analysis points to a low level of gene flow between Neandertals and modern humans, perhaps 1-4\% (Green et al. 2010). Interestingly, Neandertals share more genetic variants with presentday humans in Eurasia than with those in sub-Saharan Africa (Green et al. 2010). This finding suggests that gene flow from Neandertals into the ancestors of non-Africans occurred before the divergence of Eurasian groups from each other, although other scenarios have been proposed (Green et al. 2010). This genetic exchange could possibly have occurred as modern humans first moved into the Middle East before 100,000 years ago, when Neandertals existed in the same region after this time, and probably until 50,000 years ago (Shea 2003b, Hublin 2009). 
As for our own species, various lines of evidence indicate that anatomically modern humans arose in East Africa some 200,000 years ago. The dating of the Omo fossils from Ethiopia indicates the antiquity of early anatomically modern $H$. sapiens at 195,000 \pm 6,000 ybp (McDougall, Brown, and Fleagle 2005). Genetic data also indicate similar time depth for modern humans based on mtDNA and Y-chromosome phylogenies (Chen et al. 2000, Ingman et al. 2000, Gonder et al. 2007, Behar et al. 2008). However, genetic data also demonstrate that AMH did not begin to expand out of Africa until approximately 60-70,000 years ago (Ingman et al. 2000, Forster 2004, Macaulay et al. 2005, Kivisild et al. 2006), meaning that $H$. sapiens existed in Africa for some 100-130,000 years before leaving the African continent. This expansion is also marked by the appearance of UP-like complexity in 70,000 year-old tools at MSA sites in South Africa (Henshilwood and Marean 2006, Backwell, d'Errico, and Wadley 2008), and its spread around the globe after that time (Foley and Lahr 2003, Shea 2003b).

How one makes taxonomic and evolutionary sense of this wide array of fossil hominins depends in part on one's predisposition to lump or split taxa. It is also contingent on the model of human origins and the early stages of hominin evolution that one accepts. For example, according to the linear model (Asfaw et al. 1999), the distinctive hominin anatomy evolved only once, and was followed by a ladder-like ancestor-descendant series, or anagenesis. In this model, there is little to no branching, or cladogenesis, until well after 3 mya.

In contrast, the so-called bushy model views hominin evolution as a series of successive adaptive radiations. During these moments of evolutionary diversification in response to novel circumstances, anatomical features present in these hominins may have been mixed and matched, producing the mosaic of traits seen in some fossils (Wood and Collard 1999, Leakey et al. 2001, Foley 2002). In fact, this model suggests that, due to the independent acquisition of similar shared characters (homoplasies), key hominid adaptations such as bipedalism or a large brain may have evolved more than once in the human lineage (Collard and Wood 2000).

Judging from the current fossil evidence, there may well have been considerable taxonomic diversity during hominin evolution around 5-7 mya. These species probably exhibited various and perhaps unknown combinations of hominin, chimp, and even novel features (Wood 2002, Wolpoff et al. 2002). However, due to homoplasy in these traits, certain of them may 
not be especially useful for phylogenetic reconstructions, since different species could possess these features without sharing a common ancestor (D. Lieberman 2000, 2007).

The complexity of hominin phylogeny has implications for how we model the evolution of the brain and mind, and think about the cultural and cognitive capabilities of our hominin ancestors. As indicated above, the evolution of our lineage was not likely to have followed a strict unilineal path but instead a reticulate branching process. The difficulty in finding a direct line linking earlier hominins with modern humans means that we must examine evidence for the emergence of human traits across the entire hominin lineage, and within the primate order, as well.

\section{GENETIC VARIATION IN HUMANS AND OTHER PRIMATES}

An important source of information about the evolution of modern human characteristics comes from studies of genetic variation in different primate species and, in particular, the great apes and humans. These studies, and especially those focusing on the comparison of human and chimpanzee genomes, have yielded new and fascinating insights into the phylogenetic relationships among primate species, and also the way that selection has shaped the evolution of coding sequences in these species. The detection of selection on certain loci is crucial because these changes in functional genes have likely led to the anatomical, behavioral, and cognitive differences that we see in monkeys, apes, and humans. What follows below is a summary of studies of the human and primate genomes and a discussion of their implications for the emergence of our species.

\section{Primate Cytogenetics}

Prior to the use of the polymerase chain reaction (PCR) and high throughput sequencing methods to analyze genetic variation, researchers characterized chromosomal banding patterns and compared segmental positions in different primate species to understand phylogenetic and functional differences between them. These structural features of chromosomes revealed information about the genomic similarity of different species. These studies did not generate direct genealogical insights into the phylogenetic relationships between species as do studies of single copy nuclear genes or uniparentally inherited genetic systems, such as the mi- 
tochondrial DNA (mtDNA) or the Y-chromosome, in large part due to recombination and other rearrangements that have taken place over many millions of years. However, they demonstrated the relative similarity of the genomes of these species, and identified sorts of evolutionary processes that they have experienced over the past 10-20 million years.

In reviewing one cladogram based on chromosome banding patterns (Fig. 2.3), we can see that there have been a number of significant changes in chromosome structure over the past 14-25 million years, including fissions, fusions, and inversions. Gibbons have undergone tremendous chromosome rearrangements, and they also show a range of karyotypes, while the branch leading to the orangutan and African apes has undergone two fissions to produce the 48-chromosome karyotype. In the human lineage, there has been a fusion of two chromosomes present in chimpanzees and other primates to create the human chromosome 2 , thereby reducing the human karyotype to 2 n=46 (e.g., Ferguson-Smith and Trifonov 2007). A recent survey of sequence variation of the $>600 \mathrm{~kb}$ surrounding this fusion

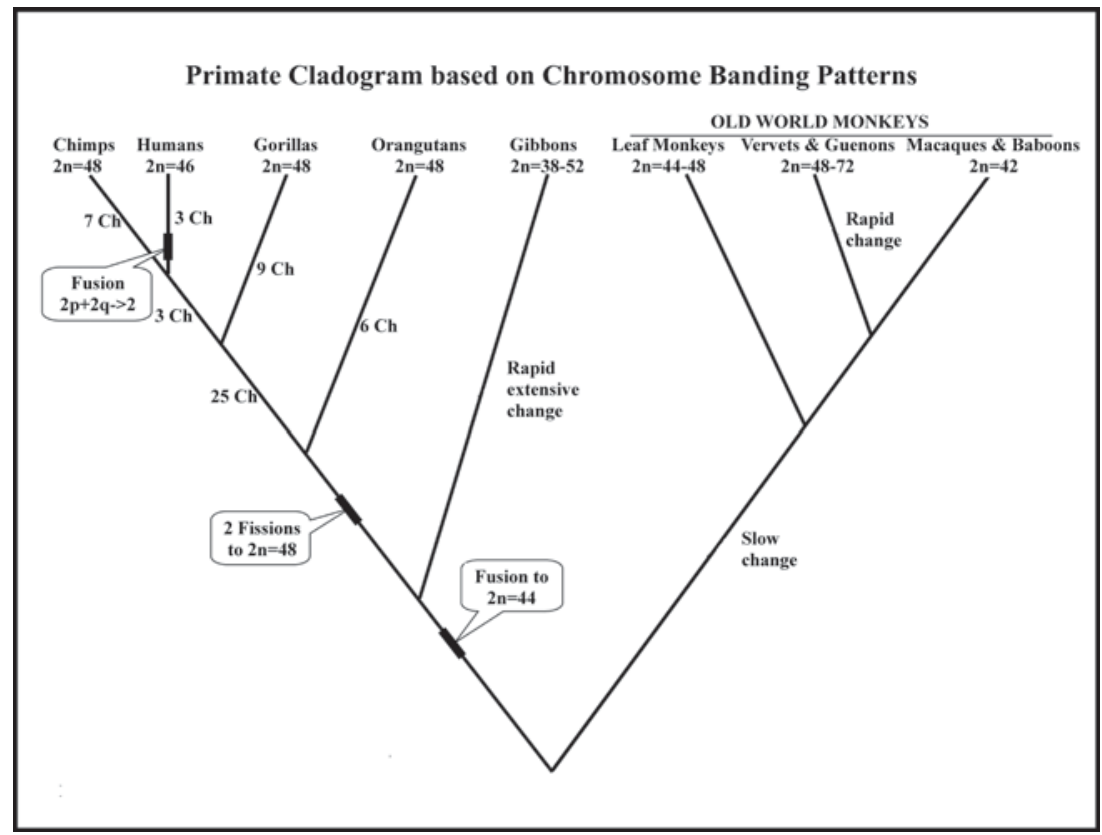

2.3. Primate cladogram based on chromosomal banding patterns. (Modified after S. Jones et al. 1992, Chap. 8.2, J. Marks, p. 301.) 
point revealed multiple duplications of large sequence blocks that originated on other chromosomes (Fan et al. 2002), some of which may be associated with breakpoints of inversions that occurred during primate evolution, or with recurrent chromosome rearrangements that have taken place in humans.

Overall, both cytogenetic and molecular data indicate that genomic rearrangements have occurred frequently during primate genome evolution and significantly contributed to the DNA differences between these species (Fan et al. 2002, Frazer et al. 2003, Ciccarelli et al. 2005, Ferguson-Smith and Trifonov 2007). Because they are often found in intergenic regions, such rearrangements may play a role in gene expression differences between humans and other primates (Frazer et al. 2003).

\section{Primate Genome Expansion}

Recent work at the genomic level has confirmed the dynamic quality of primate genomes, as well as the extent of chromosomal rearrangements and expansion implied by earlier cytogenetic work. Compared to those of other mammalian species, the genomes of humans and other primates are enriched with large, interspersed segmental duplications (SDs) with high levels of sequence identity. These SDs appear to have arisen in waves of duplication during different phases of primate evolution. These genomic comparisons also point to a strong association between duplication, genomic instability, and large-scale chromosomal rearrangements. More important from an evolutionary standpoint, the occurrence of these SDs may have generated novel primate gene families, as well as significantly influenced human genic and phenotypic variation (Bailey et al. 2002, Bailey and Eichler 2006, Eichler 2006) (Fig. 2.4).

Why is this feature of SDs so important? Compared to single copy nuclear genes, SDs are 5-10 times as likely to show interspecies and intraspecies structural and/or copy-number variation (Fortna et al. 2004, Cheng et al. 2005, Tuzun et al. 2005). These copy number variants (CNVs) have, in turn, been correlated with differences in mRNA expression levels, and, thus, appear to exert an influence on normal gene regulation (Hollox, Armour, and Barber 2003, Khaitovich et al. 2004, Cheng et al. 2005). Strong signatures of positive selection are also common in segmentally duplicated genes (Johnson et al. 2001, Semple, Rolfe, and Dorin 2003, Birtle, Goodstadt, and Ponting 2005, Chimpanzee Sequencing and Analysis Consor- 

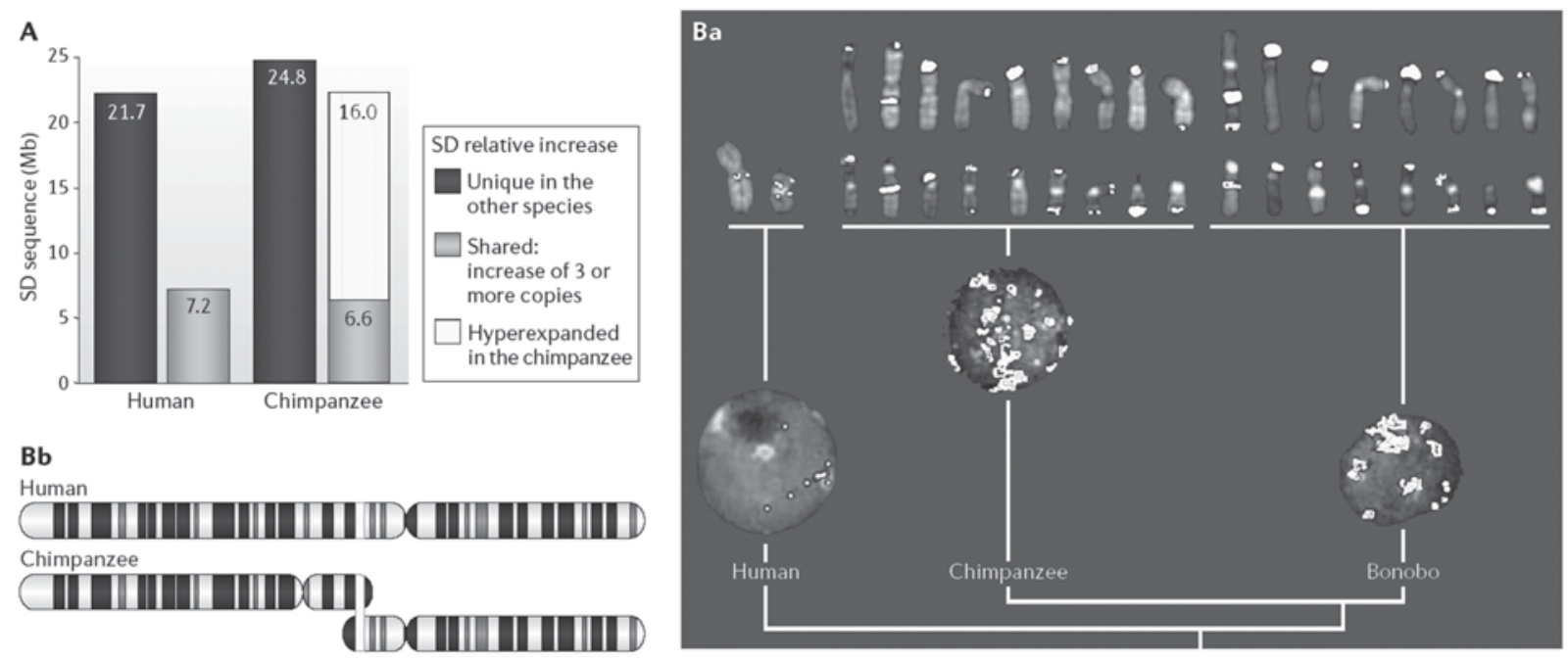

2.4. Variation in genomic segmental duplication (SD) content between chimpanzees and humans. Panel A: A comparison of duplicated sequence from the chimpanzee and human genomes allowed the identification of regions of shared duplication and those that contain human-specific or chimpanzee-specific multicopy sequence ( $>94 \%$ identity and $>20 \mathrm{~kb}$ ). An estimated $60 \%$ represented duplicative gain, whereas $40 \%$ of the change occurred as a result of deletion of ancestral duplications. Overall, as shown in the graph, a minimal estimate of $76 \mathrm{Mb}$ is differentially duplicated between humans and chimpanzees, corresponding to 3-5 Mb duplication gain per million years. Panel B: Hyperexpansion of an SD in the chimpanzee lineage. Fluorescence in situ hybridization staining (white) is shown in panel Ba for an SD duplicon of $\sim 40 \mathrm{~kb}$, revealing an expansion to 400-500 copies of this sequence in the chimpanzee and bonobo, mainly near telomeres (only chromosomes that carry the duplicon are shown). This expansion added $\sim 16 \mathrm{Mb}$ of duplicated sequence in a common ancestor of chimpanzees and bonobos that is not present in gorillas and humans. As shown in panel $\mathbf{B b}$, the same SD underlies an association between duplication and rearrangement: it is associated with a large-scale chromosome fusion event that produced human chromosome 2 (this chromosome is shown next to the two orthologous chimpanzee chromosomes; the SD lies $40 \mathrm{~kb}$ proximal of the fusion point). (Reprinted with permission from Macmillan Publishers Ltd: Nature Reviews Genetics 7 (2006), J. Bailey and E. Eichler, pp. 552-64, Fig. 5.) 
tium 2005, Ciccarelli et al. 2005).

Furthermore, it has been noted that several functional categories are enriched among these genes, including loci involved in immune response, xenobiotic recognition (e.g., olfactory reception), and reproduction (Clark et al. 2003a). These findings suggest a very important role for SDs in primate and human adaptive evolution. In support of this view, polymorphic insertions, deletions, and inversions in humans are non-randomly distributed, with a 4-12-fold greater frequency near sites of SD (Sebat et al. 2004, Iafrate et al. 2004, Feuk et al. 2005, Sharp et al. 2005, Tuzun et al. 2005, Eichler 2006). Similarly, in chimpanzee populations there is an almost 20fold enrichment of copy-number variation for regions in which duplications arose in the human-chimpanzee ancestor (Bailey and Eichler 2006, Perry et al. 2006, 2008). These data further indicate that duplicated regions are continuing to rearrange and evolve in contemporary primate populations.

The human genome has also grown in size over the past 50 million years through retrotransposition (Liu et al. 2003). Retrotranspositions are a subclass of transposons, or jumping genes, that can amplify themselves in a genome through self-replication. They are ubiquitous components of the DNA of many eukaryotic organisms, and comprise around $42 \%$ of the human genome (Lander et al. 2001). While rates of single-nucleotide substitution remain relatively constant in different primate lineages, those for retrotransposition have not. These evolutionary differences have led to a 15-20\% expansion of the human genome size over the last 50 million years of primate evolution, $90 \%$ of it being attributable to new retroposon insertions. Within the last 6-8 million years, the human chromosomes have expanded 30 megabases $(\mathrm{Mb})$ compared to the primate genome of the chimpanzee (Liu et al. 2003). In fact, it appears that our genome continues to significantly expand due to shifts in retrotranspositional activity. The exact implications of this chromosomal expansion for selection and adaptation in the human and chimp lineages have yet to be fully elaborated.

\section{Nuclear Gene Variation}

Much work has been done with functional genes in humans, apes, and monkeys to clarify their evolutionary relationships prior to the emergence of genomic sequences. Since that time, data obtained from comparative and functional genomics has elaborated on the initial findings obtained through traditional sequencing and chromosomal mapping approaches. 


\section{PRIMATE NUCLEAR GENETIC TREE}

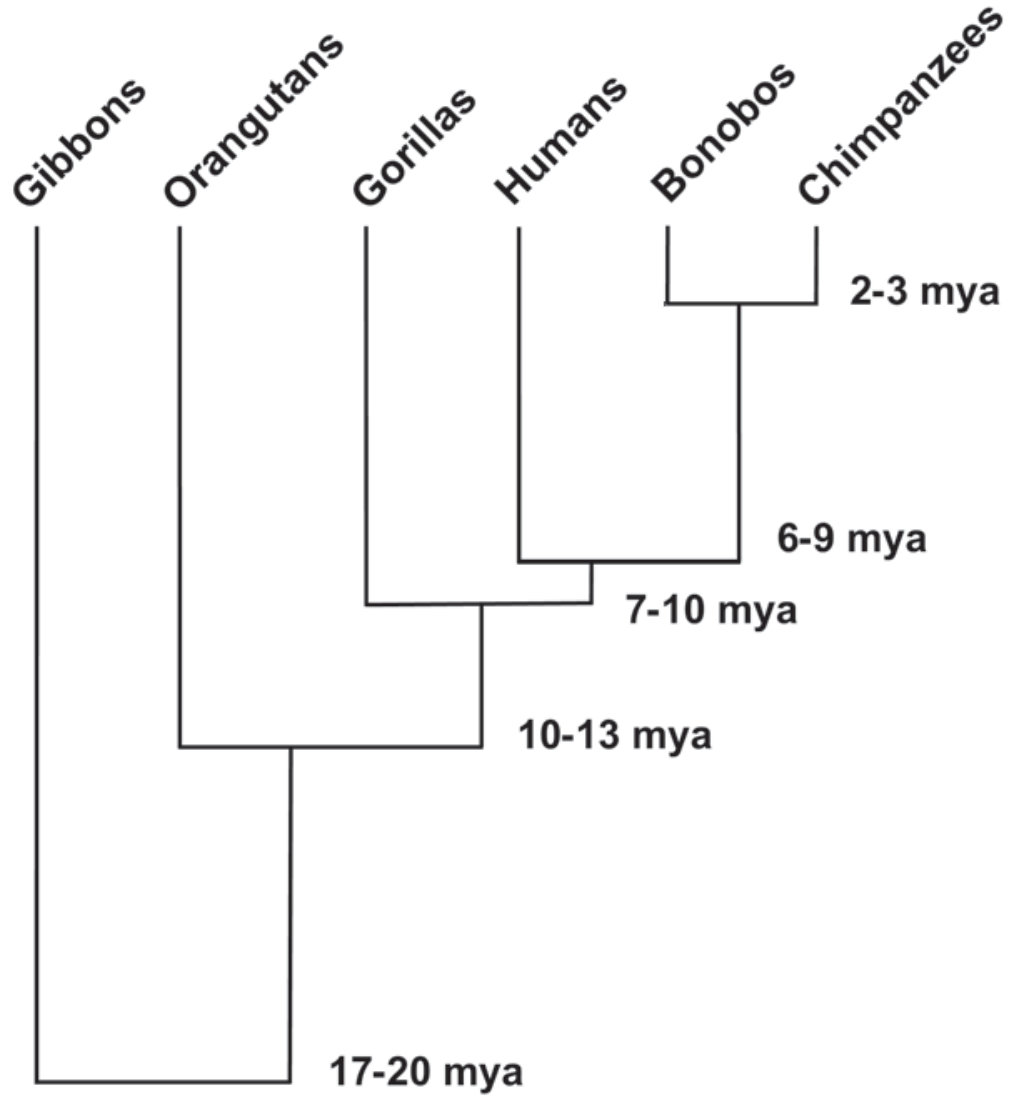

2.5. A phylogeny showing the evolutionary relationships between different hominoid species. The numbers indicate estimated millions of years (myr) from the last branch point to the present along particular lineages. The time estimates are based on both fossil evidence and genetic data from the species represented in the tree. (After Klein and Takahata 2002.)

Various nuclear gene sequences have been used to reconstruct phylogenetic relationships of humans and hominoid ape species. In the phylogeny shown in Figure 2.5, there is a clear separation of the human-chimp lineage from that uniting gorilla with human-chimp. The human-chimp split dates to 5.4 mya and human-gorilla to 7.3 mya, while the common chimpbonobo split occurred around 2.5 mya. The human-chimp split is somewhat later than other estimates of the genetic divergence of these species (e.g., 
Arnason et al. 2000), but is consistent in showing a shallower subdivision of these hominoid lineages than was once supposed based on morphological and fossil evidence (Klein and Takahata 2002).

These kinds of phylogenetic reconstructions are now being investigated at the genomic level through sequence comparisons involving multiple different primate species. In one such study of four genomic regions from human, chimp, gorilla, and orangutan comprising $\sim 2$ million base pairs, Hobolth et al. (2007) estimate the human-chimp speciation event at $4.1 \pm$ 0.4 mya and fairly large ancestral effective population sizes $(65,000 \pm 30,000$ for the human-chimp ancestor). Interestingly, they noted that approximately half of the human genome coalesced with chimpanzee after speciation with gorilla. Through their comparison of genomic segments from humans, chimpanzees, gorillas, orangutans, and macaques, Patterson et al. (2006) suggested that the apparently short divergence time between humans and chimpanzees on the $\mathrm{X}$ chromosome was explained by an interspecific hybridization event in the ancestry of these two species. Their data also indicated that hominin-chimp gene flow ceased and final speciation occurred as recently as 4.0 mya. This date is generally consistent with the hominin fossil record, in particular, the emergence of the genus Australopithecus, and suggests that, while basal to the hominin clade, Orrorin and Sahelanthropus may not demarcate the exact starting point of the human lineage.

A similar effort to determine the genomic divergence among hominoids and estimate the effective population size of the common ancestor of humans and chimpanzees involved the analysis of 53 autosomal intergenic nonrepetitive DNA segments from a human, a chimpanzee, a gorilla, and an orangutan (Chen and Li 2001, Chen et al. 2001). The average sequence divergence was only $1.24 \% \pm 0.07 \%$ for the human-chimpanzee pair, $1.62 \%$ $\pm 0.08 \%$ for the human-gorilla pair, and $1.63 \% \pm 0.08 \%$ for the chimpanzeegorilla pair. When these DNA segments were subjected to phylogenetic analysis, they strongly supported the Homo-Pan clade. However, when each segment was analyzed separately, about $42 \%$ of them produced a phylogeny that was inconsistent with the species tree (Chen and Li 2001).

These results were interpreted as revealing a large effective population size (Ne) of the common ancestor of Homo and Pan (between 52,000 to 96,000), as also seen by Hobolth et al. (2007). Interestingly, these estimates are 5-9 times larger than the long-term effective population size of humans $(\sim 10,000)$ estimated from genetic polymorphism data and linkage disequi- 
librium studies (Zietkiewicz et al. 1998, Wall 2003, Zhivotovsky, Rosenberg, and Feldman 2003, Tenesa et al. 2007). Thus, the human lineage appears to have experienced a large reduction in effective population size after its separation from the chimpanzee lineage some 4.6-6.2 mya (Chen and Li 2001).

\section{Primate mtDNA Evolution}

Much has also been learned about primate evolution from the analysis of mtDNA. The phylogenetic analysis of hominid mtDNAs shows a clear differentiation of each species in terms of its maternal lineage(s) and differing degrees of branching within each species-specific clade (Gagneux et al. 1999) (Fig. 2.6). The hominin lineage is distinct from the chimpanzee lineage in having very short branches at its terminus and lacking any truly archaic branches, aside from that leading to Neandertals.

By contrast, chimpanzees show far greater mtDNA diversity than humans in terms of the number and antiquity of the branches at both the species and

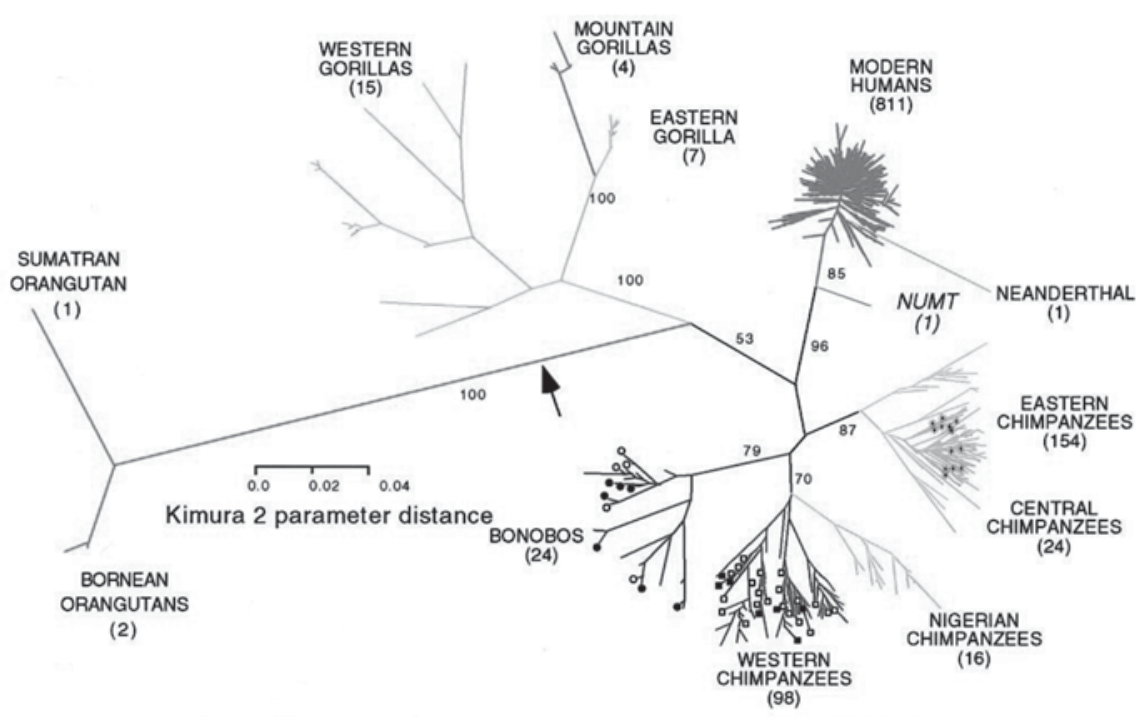

2.6. An unrooted neighbor-joining tree of hominid mtDNA sequences. The numbers of samples per species or subspecies represented in the tree are shown in parentheses. Bootstrap values $\geq 50 \%$ for the primary internodes are shown along the branches. The position of the midpoint root is indicated by an arrow. (From P. Gagneux et al. 1999:5077-82, Fig. 1. @ 1999 National Academy of Sciences, USA.) 
the subspecies levels. This pattern of branching implies long-term occupation of the species range, the maintenance of population structure over time, and the preservation of much of the genetic diversity that had accumulated over the past 3-5 million years, not to mention a larger effective population size of chimpanzees relative to hominins (Gagneux et al. 1999).

\section{FUNCTIONAL DIFFERENCES IN HUMAN-CHIMP GENOMES}

The previous discussion of genetic variation in primates and humans sets the stage for a review of analyses of human and chimp genomes, which have now been completely sequenced, at least in draft form. This analysis is facilitated by the availability of genomic sequence data from other species such as rat (Rat Genome Sequencing Project Consortium 2004), Drosophila (Adams et al. 2000), rhesus macaque (Rhesus Macaque Genome Sequencing and Analysis Consortium 2007), and others. Based on the comparison of genomic data, human and chimpanzee gene sequences are nearly $96 \%$ identical, implying close phylogenetic links between them (e.g., Clark et al. 2003a).

However, these two genomes also exhibit biologically important changes that have occurred since the ancestral lineages of these species diverged. Studies of primate genomes have identified hundreds of genes that show a pattern of sequence change consistent with adaptive evolution occurring in human ancestors. These data have also allowed researchers to estimate the extent and regional variation of the mutational forces shaping these two genomes. The patterns of evolution in human and chimpanzee protein-coding genes are also highly correlated and dominated by the fixation of neutral and slightly deleterious alleles.

Through using the chimpanzee genome as an outgroup taxon in studies of human genetic variation, researchers have been able to identify signatures of selective sweeps in recent human evolution. These selective sweeps facilitate a greater understanding of the molecular basis of adaptive evolution. As previously noted, the rapidly evolving categories of genes within the hominin lineage are primarily related to immunity and host defense, reproduction (especially spermatogenesis), and sensory perception (especially olfaction). These are the same categories that are known to be undergoing rapid evolution within the broader mammalian lineage, as well as more 
distantly related species (Chimpanzee Sequencing and Analysis Consortium 2005, Clark et al. 2003a, Sabeti et al. 2006, Nielsen et al. 2005, Williamson et al. 2007, Bustamante et al. 2005).

We also see evidence for accelerated evolution in several functional classes of genes involved in nuclear transport, cell adhesion, ion transport, and sound perception that have undergone accelerated divergence in both human and chimpanzee (Clark et al. 2003a, Chimpanzee Sequencing and Analysis Consortium 2005), while other candidates for positive selection have novel, as-yet-unknown functions (Clark et al. 2003a, Nielsen et al. 2005, Bustamante et al. 2005). There is further evidence that, in addition to being influenced by positive selection, certain genes have experienced particularly strong relaxation of constraints in hominins. On the other hand, roughly $13.5 \%$ of potentially informative loci exhibited few amino acid differences between humans and chimpanzees. This observation indicates that weak negative selection and/or balancing selection has operated on mutations at these loci (Bustamante et al. 2005).

Several studies have also noted that X-linked genes are significantly overrepresented among rapidly evolving genes in humans and chimps (Chimpanzee Sequencing and Analysis Consortium 2005, Nielsen et al. 2005). The increased selection on the $X$ chromosome is undoubtedly related to the fact that it contains a large number of sperm- and testis-associated genes (Nielsen et al. 2005), which are frequent targets of selection. In addition, the hemizygosity of the $\mathrm{X}$ chromosome in males exposes recessive alleles to selective pressure, which may further promote rapid evolution (Schaffner 2004).

Human-chimp genome comparisons have also permitted a closer examination of regions of the human genome that may reflect recent adaptive evolution. For example, a recent study of different human populations revealed regions of the human genome with strong evidence of a recent selective sweep, with the estimated position of the selective sweep falling within $100 \mathrm{~kb}$ of a known gene (Williamson et al. 2007). Within these regions, a number of loci of biological importance were identified, including ones involved in pigmentation pathways, components of the dystrophin protein complex, clusters of olfactory receptors, genes involved in nervous system development and function, immune system genes, and heat shock genes. Overall, Williamson et al. (2007) noted that as much as $10 \%$ of the human genome had been influenced by linkage to a selective sweep. 


\section{Olfaction}

Having described the overall patterns of genetic divergence and selection in the human and chimp genomes, I will now focus on several key genetic adaptations in the hominin lineage and explore their relationship to other anatomical, physiological, and behavioral characteristics.

One set of genes that has undergone very rapid change in the chimpanzee and especially the human lineage are those involved with olfaction. Nearly 50 such genes linked to smell show evidence of positive selection (relaxed selection) in humans (Chen and Li 2001, Clark et al. 2003a, Gilad et al. 2003a, 2003b, Chimpanzee Sequencing and Analysis Consortium 2005). These differences probably reflect the reduced importance of smell in the human lifestyle relative to that of chimpanzees.

One of the largest gene families influencing the sense of smell, the olfactory receptors (OR), is known to be undergoing rapid divergence in primates. OR genes are encoded by the largest mammalian gene superfamily of $>1,000$ genes (Ben-Arie et al. 1994, Gilad et al. 2003a, 2003b). Analysis of these genes in the draft assembly of the human genome has suggested that more than 100 functional human ORs are likely to be under no evolutionary constraint (Gilad, Man, and Glusman 2005).

\section{Bipedalism}

Bipedalism is a key human adaptation and a defining feature of the hominin clade. It is marked by a series of skeleto-muscular, neurological, postural, and developmental changes that permit habitual movement and balance on two legs (Lovejoy 1981, Alexander 1992, Jablonski and Chaplin 1993, Spoor et al. 1994, 1996, Fitzpatrick et al. 2006). The anatomical changes involved in supporting bipedal creatures include changing pelvic structure, angled knees, curved lumbar region, reorientation of muscle attachments, and adducted large toes, among others (Lovejoy 1981, Alexander 1992, Jablonski and Chaplin 1993). Within the hominin clade, researchers have recognized two forms of bipedalism, termed "facultative" (i.e., optional) or "obligate" (habitual) (Wood 2002). As indicated in Figure 2.2, these forms imply different degrees of adaptation to this locomotory pattern, hence, the presence of the anatomical, behavioral, and physiological changes associated with it.

The anatomical changes involved in supporting bipedalism also posed challenges to hominins that were undergoing increasing encephalization from about 2.0 mya to the present. The problem of birthing increasingly 
large-headed infants necessitated changes in the pelvic structure and shape of the birth canal, which, in turn, created obstetric complications (Trevathan 1987, Whitcome, Shapiro, and Lieberman 2007). The trade-off between being fully bipedal and becoming larger brained resulted in delayed prenatal development and significant post-natal brain growth in hominin offspring (Bogin 2001, Aiello and Wells 2002). It also appears that the growth in size and complexity of neuronal connectivity in the human cerebellum compared to chimps may be related to upright posture and modulation of muscle movements and timing, not to mention aspects of language processing (Gazzaniga, Ivry, and Mangun 1998, Schoenemann 2006). Therefore, in addition to being an adaptation with important anatomical and physiological consequences, bipedalism also likely had an important influence on the development of human cognitive abilities.

Comparative genomic work has indicated that certain selective changes may have occurred in genes that influenced the evolution of bipedalism. These include loci that may be involved in long-bone growth (cytoskeletal proteins) and hair loss (thermoregulation) (Winter et al. 2001, Clark et al. 2003a), as well as other loci involved with actin binding, cytoskeletal formation, and ectoderm development and which may be under weak negative selection (Clark et al. 2003a, Bustamante et al. 2005). Cytoskeletal protein genes in particular show extensive amino acid polymorphism within humans but limited amino acid divergence between humans and chimpanzees (Bustamante et al. 2005).

These new genetic data provide a new context in which to understand the emergence of bipedalism in the hominin lineage based on fossil evidence. To date, Sahelanthropus is only known from craniodental evidence (Brunet et al. 2002). Therefore, while the position of its foramen magnum suggests that it was bipedal (Zollikofer et al. 2005), postcranial fossils from this taxon are needed to confirm its bipedal status. Fossil femora discovered in the Lukeino Formation of Kenya and attributed to Orrorin tugenensis (Senut et al. 2001) purportedly provide the earliest postcranial evidence of hominin bipedalism at 6 mya (Pickford et al. 2002). However, there has been considerable debate over the functional and phylogenetic significance of these femora in relation to those of other hominin forms (e.g., Begun 2004).

Subsequent analysis of hominin and ape femora has provided additional insights into the emergence of bipedality in the hominin lineage (Richmond and Jungers 2008). This study has shown that the O. tugenensis femur differs 
from those of apes and Homo, and most strongly resembles those of Australopithecus and Paranthropus. Femoral morphology also indicates that $O$. tugenensis shared distinctive hip biomechanics with australopithecines. This evidence suggests that the morphological complex involving the pelvis and femora evolved early in hominin evolution and persisted for almost 4 million years until additional modifications of the hip appeared in early Homo.

However, the similarities in femoral morphology among the early hominins do not exclude the possibility that they also engaged in other forms of locomotory behavior. In fact, the upper limb fossils of $O$. tugenensis exhibit morphological features related to arboreal climbing, such as curved proximal manual phalanges (Senut et al. 2001). The presence of these features in a species close to the chimp-human split implies that bipedalism evolved from a hominin ancestor that was adapted to orthograde and vertical climbing. This interpretation, in turn, indicates that the ancestor had a climbing and knuckle-walking repertoire (Richmond, Begun, and Strait 2001) rather than being an orangutan-like arboreal specialist (Thorpe, Holder, and Crompton 2007).

The comparative biomechanical anatomy of $O$. tugenensis femora suggests that this species is a basal hominin adapted to bipedalism (Senut et al. 2001, Richmond, Begun, and Strait 2001, Thorpe, Holder, and Crompton 2007). In addition, current evidence suggests that an Australopithecus-like bipedal morphology evolved early in the hominin clade and persisted successfully for most of human evolutionary history. However, truly obligate bipedalism apparently did not arise until the emergence of Homo erectus, in contrast with the facultative bipedalism of early hominids. Regardless of the exact time when obligate bipedalism took root in the hominin lineage, the anatomical changes underlying this locomotory adaptation were substantial (Fig. 2.7).

There is also the matter of the bioenergetics of bipedal locomotion. Various studies have attempted to quantify the energetic costs of quadrupedal versus bipedal locomotory patterns in humans, hominins, and chimps to determine whether bipedality conferred some benefit to those species that habitually employed this pattern of movement (Pontzer and Wrangham 2004, Steudel-Numbers 2006, Sockol, Raichlen, and Pontzer 2007). One such study examined stride lengths, speed, and energy costs in the walking of Australopithecus afarensis, based on the Laetoli footprints. These investigators noted that, by 3.5 mya, at least some early hominins, despite their small stature, could sustain efficient bipedal walking at absolute speeds within the 

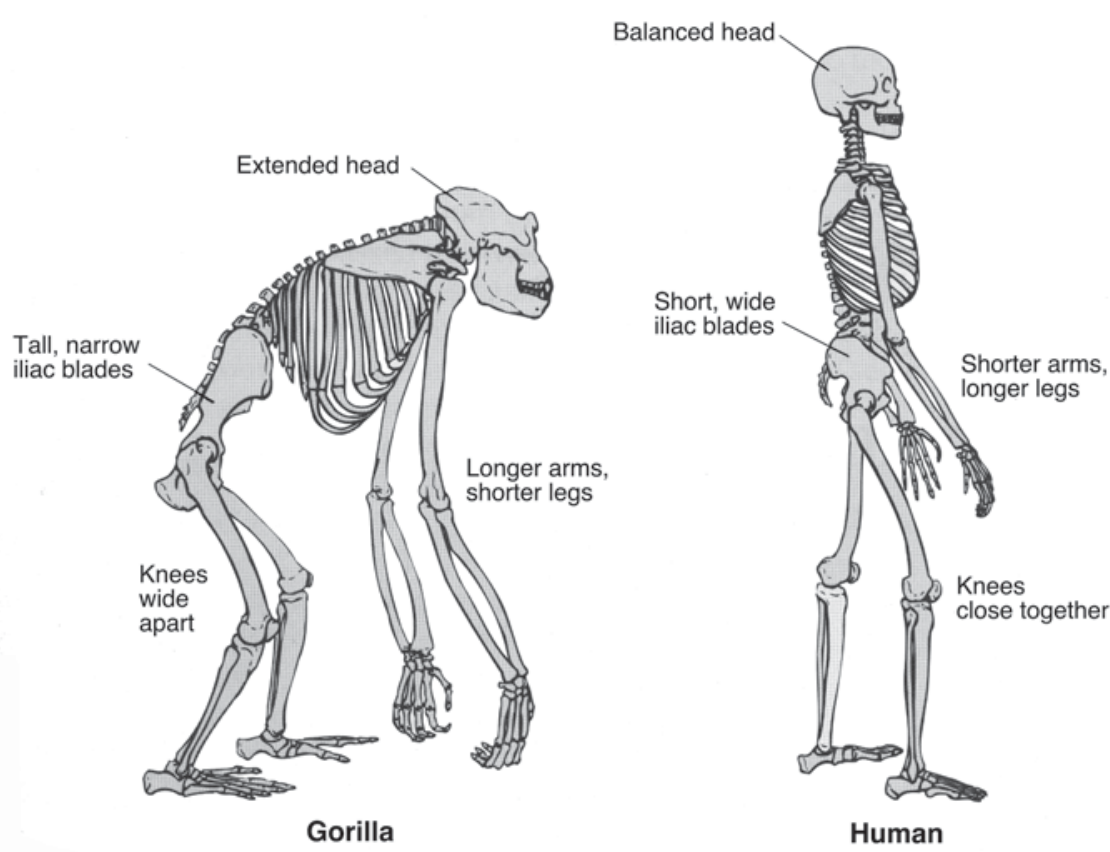

2.7. Human bipedality and ape anatomy. A comparison of gorilla and human anatomy and stature. (From Bernard G. Campbell, James D. Loy, and Kathryn CruzUribe, Humankind Emerging, 9th ed., ( ) 2006, Fig. 13.16. Printed and electronically reproduced by permission of Pearson Education, Inc., Upper Saddle River, New Jersey.)

range shown by modern humans (Sellers et al. 2005).

Walking on two legs also produced certain thermoregulatory demands that had to be compensated for physiologically. The need for hominids to walk long distances upright probably led to the loss of much of their body hair and the emergence of an extensive system of sweat glands to remove excess heat through transpiration (Wheeler 1984, 1991), and perhaps also darker skin pigmentation to protect the skin from excessive UV light (Jablonski 2004). In fact, according to the thermoregulatory model, the increased heat loss, increased cooling, reduced heat gain, and reduced water requirements conferred by a bipedal stance in a hot, tropical climate was the primary selective pressure leading to bipedalism (Wheeler 1991). However, others have suggested other possible selective forces, such as ecological adaptations for feeding (Hunt 1996) or the social, sexual, and reproductive conduct of early hominids (Lovejoy 1981). In any case, the shift from a qua- 
drupedal to a bipedal locomotory adaptation led to a major transformation of hominins at anatomical, behavioral, cognitive, and physiological levels.

\section{Dietary Changes}

Comparisons of the human and chimp genomes have also indicated that nearly 80 genes used to digest proteins also differ between these two species (Clark et al. 2003a, Chimpanzee Sequencing and Analysis Consortium 2005). These differences likely reflect how the human diet has changed in the 5 million years since hominins split from chimpanzees. The most significant dietary change occurring during this time period was the greater reliance on meat in the diet, which is evident in the taphonomic record for early Homo (Stanford 2001, Foley 2002, Dominguez-Rodrigo and Pickering 2003). This evidence marks a major shift in subsistence strategy to one focusing on acquisition of fats and proteins in meat, whether through scavenging or hunting (Stiner 2002).

The transition to a diet consisting of greater amounts of meat and fats is also reflected in changes in gut morphology in apes and hominins (Aiello and Wheeler 1995, Milton 1999) (Fig. 2.8). Concurrent with increasing encephalization in the hominin lineage, within the genus Homo (see below), the hominin digestive system also underwent a major transformation. Chimps (and most probably early hominins) have a longer large intestine and a shorter small intestine, as needed by an omnivorous species with a significant intake of vegetative matter and fibrous food sources. By contrast, humans have the opposite configuration, a shorter large intestine and longer small intestine, allowing them to absorb more of nutrients, minerals, and fats in the food sources that they consume (Aiello and Wheeler 1995, Milton 1999). This remodeling of the body had significant bioenergetic implications, as both the brain and the stomach are metabolically costly organs, although it was possibly mediated by changes in the proportion of body weight comprised of fat (Milton 1999, Aiello and Wells 2002). Overall, $H$. erectus exhibits features more similar to those of modern humans than those of earlier and contemporaneous australopithecines and paranthropines, such as larger relative brain sizes, larger bodies, higher energetic needs, slower rates of growth and maturation, obligate bipedalism, and smaller teeth and jaws (Aiello and Wells 2002, Antón and Swisher 2004).

There has been considerable effort to understand the conditions under which these morphological and dietary changes took place. According 


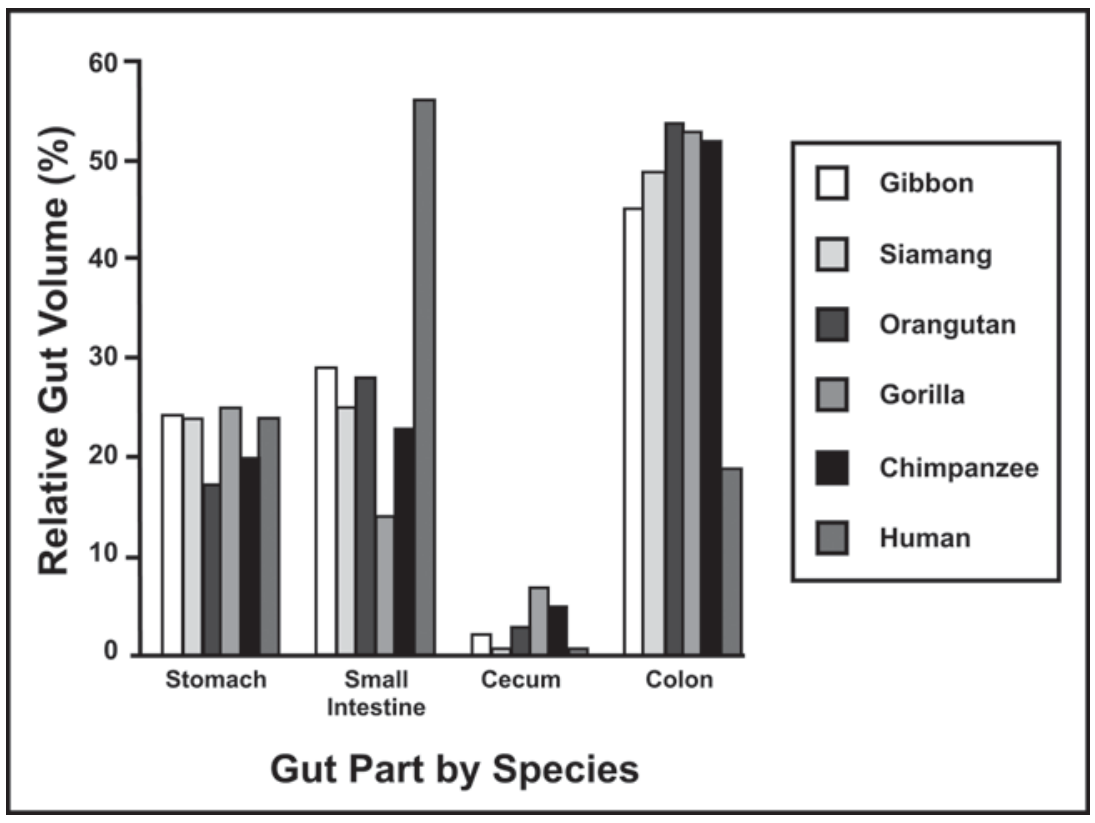

2.8. Relative gut volume proportions for some hominoid primate species. (Redrawn after Milton 1999:488-98, Fig. 1.)

to the turnover-pulse hypothesis, the emergence of the genus Homo was linked to climatic fluctuations and habitat disruption about 2.5-2.0 mya, which coincided with the initial transition of forests to open grassland in Africa (Stanley 1992, Vrba 1994, deMenocal 1995, Larick and Ciochon 1996). Under these conditions, long-term directional selection related to lower temperatures and increased aridity may have favored ecological generalization in early hominins (Potts 1998). In addition, the emergence of numerous savannah-adapted antelope species during this time would have provided diverse food resources for hominin scavengers, who were beginning to exploit prey taken by large cats and hyenas (Vrba 1985, Larick and Ciochon 1996, de Heinzelin et al. 1999, Teaford, Ungar, and Grine 2000).

Others view the role of dietary change in the origin and early evolution of the genus Homo in Africa slightly differently. For example, based on craniodental remains of early Homo, Ungar, Grine, and Teaford (2006) see no simple transition from an australopithecine to a Homo grade of dietary adaptation, or from closed forest plant diets to reliance on more open-country 
plants or animals. In their view, early Homo species more likely had adaptations for flexible, versatile subsistence strategies that would have served them well in the variable paleoenvironments of the African Plio-Pleistocene. This view is echoed by Wood and Strait (2004), who suggest that it was the dietary and behavioral flexibility exhibited by early Homo, not the narrowing of its ecological niche that enabled this lineage to persist.

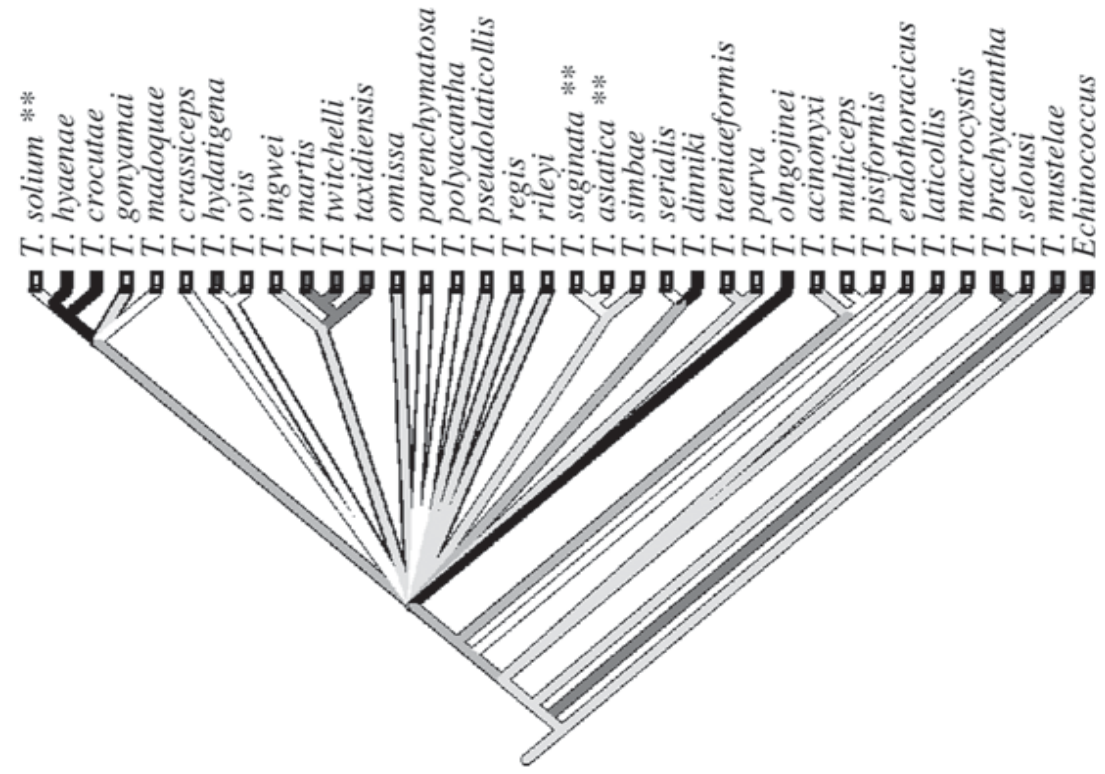

\section{DEFINITIVE HOST (unordered)}

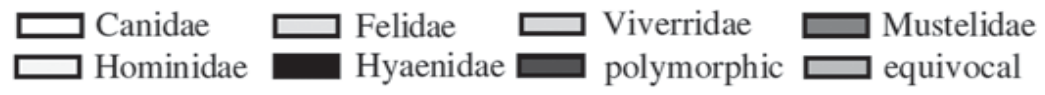

2.9. Phylogenetic relationships and host associations among species of Taenia (tapeworms). The majority rule consensus tree is based on analyses of 35 specieslevel taxa. Species of Taenia in human definitive hosts (asterisks) can be divided into two sub-clades: the "T. solium sub-clade" containing $T$. solium, $T$. hyaenae, $T$. crocutae, T. gonyamai, and T. madoquae; and the "T. saginata sub-clade" including $T$. saginata and its sister species, T. asiatica and T. simbae. Parsimony mapping was used to examine the putative relationships for Taenia spp. and definitive hosts. Not all host families are depicted on the tree due to multiple host groups and polymorphic coding in terminals for some species of Taenia (i.e., Suidae, Procaviidae, Canidae and Primates for T. solium). (From Hoberg et al., Out of Africa: Origins of the Taenia Tapeworms in Humans, PRSB 268 (2001): 781-87, Fig. 1a; used with permission.) 
We also have indirect evidence for this shift in subsistence strategy to one focusing on acquisition of fats and proteins in meat, whether through scavenging or hunting. This evidence comes in the form of morphological and genetic data from different species of tapeworms (genus Taenia). Based on these data, it appears that these parasites colonized early hominins at least twice around 2.0-2.5 mya during hominins' dietary and behavioral shifts, from herbivory to scavenging and carnivory in the Plio-Pleistocene (Hoberg et al. 2001) (Fig. 2.9). Phylogenetic analysis indicated that the immediate ancestors of these tapeworms used carnivores (hyaenids, canids, or felids) as definitive hosts and bovids as intermediate hosts.

At a behavioral level, the practice of scavenging and hunting implies the coordination of group actions to procure food sources. Some form of communication must have been involved in these activities, particularly since they involved either chasing felids and hyenids away from freshly killed animals, or stalking herd animals that were highly mobile. In this regard, comparisons between chimp and human hunting practices reveal considerable differences between them, including the types of prey taken, the coordination of group action, and the sharing of meat after an animal is killed (Stanford 2001). The lack of coordinated group actions in chimps implies that the hominin form of scavenging, and later hunting, probably began during the Plio-Pleistocene period (Foley 2002, Stiner 2002, Dominguez-Rodrigo and Pickering 2003).

\section{EVOLUTION OF LANGUAGE}

What do we mean when we say language evolution? How different are language abilities between apes and humans? To begin with, humans have voluntary control of the voice, face, and hands, whereas chimps and monkeys show deliberate control only of their hands. Both humans and apes are capable of learning and imitation, but only humans can clearly teach others how to do tasks, while apes require human training to repeatedly perform imitative behaviors. While great apes can clearly master the use of hundreds of symbols and rudimentary grammar, humans are capable of learning a far greater vocabulary and symbolic repertoire (Premack 2004). In addition, humans have both recursive and non-recursive theories of mind, and the capacity to understand recursive and non-recursive grammars, whereas apes or monkeys are limited to non-recursive grammars (Premack 2004). Moreover, as P. Lieberman (2007:39) notes, "speech also requires a brain 
that can 'reiterate' or freely reorder a finite set of motor gestures to form a potentially infinite number of words and sentences." This appears to be lacking in chimps and other nonhuman primates.

While there must be a genetic basis to the differences in cognitive and language abilities observed in different primate species, we have also learned much about them through the analysis of brain anatomy, neuronal complexity, and vocal tract anatomy in these same taxa. Whereas language abilities were once thought to reside mostly in Broca's and Wernicke's areas in the left hemisphere of the brain, we now understand that many different parts of the brain are involved in speech production and comprehensive and symbolic behavior. Comparative studies of primate and human brain anatomy viewed in the context of new genetic data have also given us insights into aspects of brain growth and development. Furthermore, research on genes affecting brain size and studies of neuronal connectivity and morphology suggests different kinds of wiring in primate and human brains.

\section{Language, Hearing, and Vocal Apparatus}

Human-chimp genome comparisons have identified changes in 21 human genes that are linked to hearing. Such genes not only enable humans to understand speech in the brain, but also are involved in hearing and are likely related to the linguistic abilities of modern humans. One particular locus, the alpha tectorin gene, shows considerable differences between chimps and humans. Interestingly, this locus is expressed in the brain and in the hearing apparatus itself in a membrane of the inner ear (Clark et al. 2003a). Its importance for hearing had previously been known, as mutations in this gene cause congenital deafness in humans (e.g., Verhoeven et al. 1998).

Language or speech production also relies on having the appropriate anatomical features of vocal apparatus. As noted in Figure 2.10, humans and apes differ considerably in the size and shape of the vocal tract, the position of the tongue in the oral cavity, and the location of the larynx in the throat. In apes, we observe the higher position of the larynx, a longer and shallower tongue and oral cavity, and the inability to use tongue and oral cavity to produce vowel sounds. By contrast, the human tongue's shape and position creates 1:1 oral-to-pharyngeal proportions of the supralaryngeal vocal tract. Overall, "the chimpanzee lacks a vocal tract capable of producing the 'quantal' sounds (regions of acoustic stability) that facilitate both speech production and perception, and a brain that can reiterate the pho- 

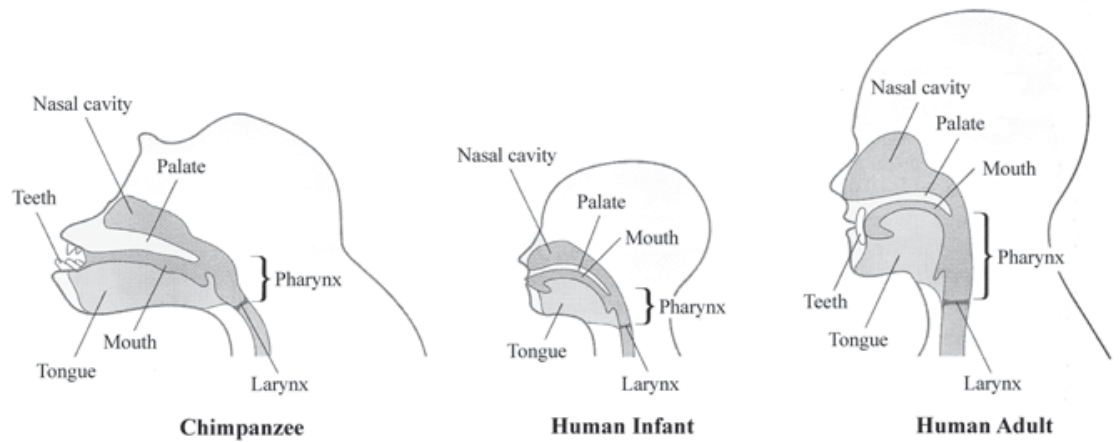

2.10. A comparison of chimpanzee and human vocal anatomy. (From Bernard $G$. Campbell, James D. Loy, and Kathryn Cruz-Uribe, Humankind Emerging, 9th ed., () 2006, Fig. 13.5. Printed and electronically reproduced by permission of Pearson Education, Inc., Upper Saddle River, New Jersey.)

netic contrasts apparent in its fixed vocalizations" (P. Lieberman 2007:39).

There has been considerable interest in understanding the ontogenetic aspects of language abilities in humans and apes. One possibility is that the developmental sequence that gives rise to the adult human vocal apparatus follows a general ape trajectory until shifting into a more human track. In this model, the human child has its larynx situated higher in its throat like apes until 2-3 years of age, when it begins producing articulate speech, at which time several developmental changes take place, including the descent of the larynx into its adult position in the throat. Thus, both anatomical and cognitive changes mark the emergence of the human ability to comprehend and produce semantically meaningful sounds (P. Lieberman 2007).

While this scenario may be generally accurate, a recent study of the ontogenetic trajectories of craniofacial traits for humans and apes revealed the existence of developmental differences even earlier in life. In this analysis, Mitteroecker et al. (2004) carried out a geomorphometric analysis of 96 traditional three-dimensional landmarks and semi-landmarks on the face and cranial base from adult $H$. sapiens, Pan paniscus, Pan troglodytes, Gorilla gorilla, and Pongo pygmaeus. They observed that $H$. sapiens specimens were clearly separated from the great apes in shape space and size-shape space. At birth, $H$. sapiens infants were already markedly different from the great apes, which overlap at this age but diverge among themselves post-natally. Hence, there are distinct ontogenetic trajectories in humans and apes that begin 
early in development and cannot be explained as differences arising through heterochrony (changes in timing in the appearance of these features).

\section{FOXP2 Gene and Language Abilities}

Additional clues about the evolution of language abilities in humans come from genetic studies of loci that are involved in the production and comprehension of speech. Many of these genes have been identified through studies of speech and language comprehension deficits in different human populations. The mutations underlying these disorders likely influence the areas of the brain that are most centrally involved in speech and language processing, namely Broca's and Wernicke's areas.

Broca's area, located in the inferior frontal gyrus (IFG) of the human brain, is one of several critical regions associated with the motor planning and execution of language. Wernicke's area, located on the posterior section of the superior temporal gyrus, on the Sylvian fissure, is known to be involved in the comprehension of spoken language. Based on current evidence, the neural circuits linking these cortical regions with the basal ganglia and other subcortical structures help to regulate motor control, including speech production, as well as syntax and other cognitive processes (P. Lieberman 2007).

Recent studies with nonhuman primates have revealed the importance of these brain areas for communication in both ape and monkey species. For example, PET scans of chimpanzees engaged in vocalization showed activity in the IFG as well as other cortical and subcortical regions (Taglialatela et al. 2008). These findings were taken as implying that the neurological structures underlying language production in the human brain may have been present in the common ancestor of chimps and humans. Related studies with monkeys have shown that the homolog of Broca's area in monkey brains corresponds to a region involved in the control of the monkey's face, larynx, tongue, and mouth (Petrides, Cadoret, and Mackey 2005, Gil-da-Costa et al. 2006). In addition, the homologous Wernicke's area was involved in the recognition of sound sequences and calls of other monkeys (Gil-da-Costa et al. 2006). However, as noted above, humans' ability to develop articulate speech relies on capabilities such as fine control of the larynx and mouth that are absent in apes and monkeys (Premack 2004, P. Lieberman 2007).

Several years ago, researchers identified a gene that is central to the human ability to develop language, due to its role in governing the embryonic development of subcortical structures of brain involved with speech 
production and comprehension. They noted that a point mutation in the forkhead-box P2 transcription factor gene (FOXP2) co-segregated with a disorder in a family in which half of the members have severe articulation difficulties accompanied by linguistic and grammatical impairment (Specific Language Impairment Consortium 2002). The predominant features of the FOXP2 phenotype of affected individuals were an impairment of both the selection and sequencing of fine orofacial movements underlying fluent speech, and the linguistic processing for both spoken and written language (Enard et al. 2002, Specific Language Impairment Consortium 2002, Groszer et al. 2008). Two functional copies of FOXP2 were required for the acquisition of normal spoken language, as the disease appears to be caused by the haploinsufficiency of gene expression. ${ }^{7}$ However, it should also be noted that other genes may also be involved in language production, such as loci on chromosomes 16 and 19 (SLI Consortium 2002).

Because of this gene's significant impact on speech production and language comprehension, particularly on fine orofacial movements that are typical of humans and not great apes, there was speculation about its possible role in the emergence of the human capability to develop proficient spoken language (Enard et al. 2002). Were this true, the time at which this FOXP2 variant became fixed in the human population could be linked to the evolution of human language.

To investigate this question, Enard et al. (2002) sequenced the FOXP2 gene in humans, a number of nonhuman primate species, and the mouse as a mammalian outgroup. They then analyzed the phylogenetic relationships among these sequences. They observed that nearly all mutations in this gene were synonymous nucleotide changes, with the branches leading to the orangutan and the mouse each having a single non-synonymous mutation (Fig. 2.11). By contrast, the human lineage exhibited only two mutations, both of which are non-synonymous changes. Enard and colleagues concluded that this gene has been the target of selection during recent human evolution, although they were not able to exclude the possibility that relaxation of constraints on FOXP2 specific to the human lineage produced this pattern of nucleotide changes (Enard et al. 2002). Their data also suggested that the fixation of the mutations in the human form of the gene occurred during the last 200,000 years of human history, that is, concomitant with or subsequent to the emergence of anatomically modern humans. These findings suggest that expansion of modern humans was 


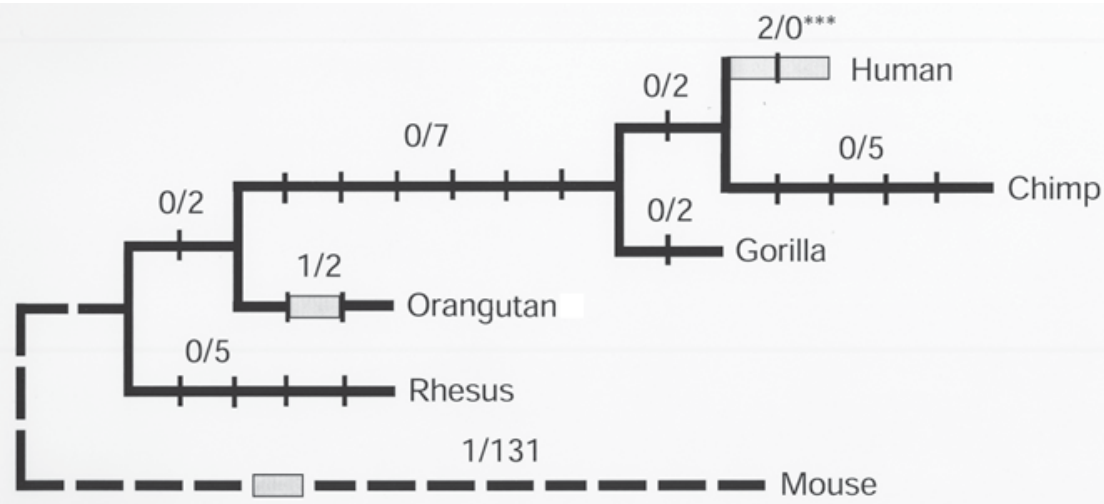

2.11. A phylogeny of the FOXP2 gene in primate species. Silent and replacement nucleotide substitutions are mapped onto this phylogeny. The horizontal bars represent nucleotide changes, while the gray bars indicate amino-acid changes. (Reprinted with permission from Macmillan Publishers Ltd: Nature 418 [2002], Enard et al., pp. 869-72, Fig. 2.)

stimulated by the emergence of a more-proficient spoken language.

Yet, while these non-synonymous changes were clearly unique to the human lineage compared to apes, it is possible that other hominin species had the same allelic variants in this gene, hence, shared a common capacity for speech production. Recent genomic sequencing of a Neandertal specimen (Green et al. 2006) has provided huge amounts of autosomal data that allow this possibility to be tested. In their examination of these data, Krause et al. (2007a) observed that the Neandertal individual shared the two mutation changes in the FOXP2 gene that are implicated in the development of speech and language in modern humans, and that these mutations appeared on the widespread modern human haplotype.

How did modern humans and Neandertals come to share the same FOXP2 haplotype? There are at least three different scenarios that can explain these findings. One is that the positively selected FOXP2 haplotype could have been transferred into Neandertals from modern humans or vice versa through gene flow. This model is now tentatively supported by genomic data from these hominins (Green et al. 2010), although mtDNA (Caramelli et al. 2003, Serre et al. 2004) and Y-chromosome (Krause et al. 2007a) data for modern humans and Neandertals reveal no gene flow between them.

Alternatively, the FOXP2 haplotype could have been present in the 
ancestral population of modern humans and Neandertals and then was positively selected in humans after their divergence from Neandertals. In this case, the relevant haplotype must have been present at a considerable frequency in the ancestral population in order to occur at relatively high frequencies in Neandertals. However, if alleles or haplotypes grow to high frequencies before being positively selected, it becomes difficult to detect a genetic signature of selective sweep (Wall and Przeworski 2000). Thus, this scenario seems implausible, as well.

The most likely scenario is that the selective sweep started prior to the divergence of the ancestral populations of Neandertals and modern humans around 300,000-400,000 years ago (Fay and $\mathrm{Wu} 2000$ ). Assuming a human-chimp divergence of 6.5 mya, the fixation of the sweep would have occurred within the last 260,000 years (Enard et al. 2002). Hence, based on these findings, it would appear that Neandertals were capable of some kind of language, although how similar it was to that of modern humans is, of course, entirely speculative.

As it turns out, the FOXP2 transcription factor is also extremely similar in many vertebrate species, showing conserved expression in neural circuits related to sensorimotor integration and motor learning (Vargha-Khadem et al. 2005, Fisher and Marcus 2006). This finding makes it possible to examine the function of this gene in other nonhuman species, and determine the phenotypes produced by mutated forms of the gene. In one such study, Groszer et al. (2008) created transgenic mice with the identical FOXP2 mutation seen in humans having specific language impairment (SLI). The resulting homozygous mutated mice showed severe reductions in cerebellar growth, but were able to produce complex innate ultrasonic vocalizations. Heterozygous mice were seemingly normal in brain structure and development and basic motor abilities, but they exhibited significant deficits in speciestypical motor-skill learning, accompanied by abnormal synaptic plasticity in striatal and cerebellar neural circuits (Groszer et al. 2008). These findings reinforce the clinical data obtained in studies of SLI patients (Specific Language Impairment Consortium 2002), and emphasize the crucial role that single genes may play in normal brain development and function.

\section{Encephalization in the Hominin Lineage}

It has long been assumed that relative brain size was a primary trait demarcating differences between humans and apes, and between the differ- 
ent hominin taxa present in the human lineage (Dunbar 1995, Kappelman 1996, Wood and Collard 1999, McHenry and Coffing 2000). Size seems to generally correlate with subsistence practices and technological shifts, as well as evidence for greater mobility, and, ultimately, symbolic behavior and language (Foley 2002, Foley and Lahr 2003, Rightmire 2004). Figure 2.12 shows the overall trend in encephalization that accompanied human brain evolution. While relative size does have some relationship to certain aspects of language ability, cultural behavior, and symbolic use (Dunbar 1995, Kappelman 1996, Wood and Collard 1999), primate species with smaller brains show some abilities to communicate, comprehend symbols, express cultural traditions, and recognize members of their social groups. Thus, both relative size and also neurological organization and development must be crucial variables in the emergence of large-brained hominins and the complex linguistic and cognitive functions that humans are able to perform.

Nevertheless, various researchers have begun searching the human

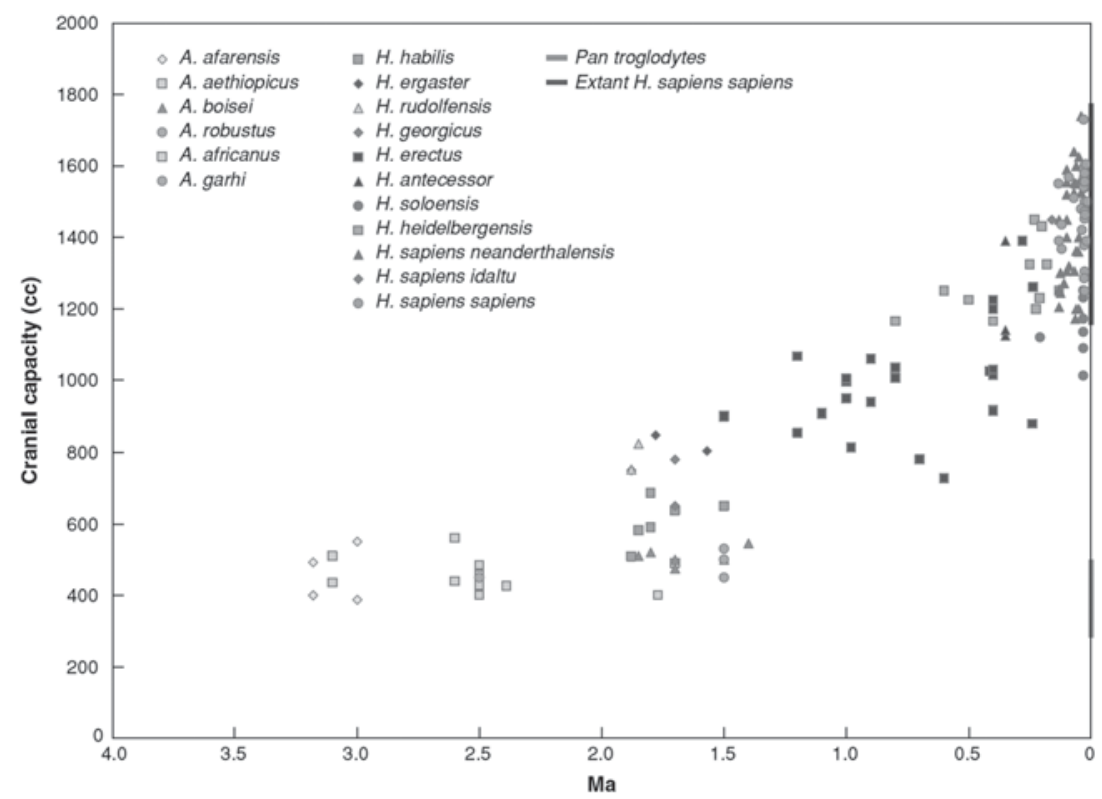

2.12. Cranial capacity in fossil hominids over time. Extant chimpanzees (Pan troglodytes) and humans (Homo sapiens sapiens) are included for comparison. Fossil data and species designations are from Holloway et al. (2004). (From Schoenemann 2006:379-406, Fig. 2; used with permission.) 
genome for genes related to brain growth and development whose sequences show evidence of positive selection in the human lineage. Such loci are viewed as potentially contributing to the emergence of modern human cognition. This search has identified two genes associated with primary microcephaly (small cerebral cortex), the first being abnormal spindlelike microcephaly associated (ASPM) and the second being microcephalin (MCPH1) (Bond et al. 2002, Evans et al. 2004a, 2004b, 2005, 2006, Kouprina et al. 2004, Wang and Su 2004, Mekel-Bobrov et al. 2007). Microcephaly reduces the human brain to $50 \%$ or less of its normal mass, i.e., to about the size of the brain of chimpanzees or early hominin ancestors.

Recent work on the microcephalin gene in humans and other primates has provided some very interesting insights into the evolution of the hominin brain. This gene is highly polymorphic in human populations, with most mutations being non-synonymous in nature (Wang and Su 2004). In fact, researchers have identified, within modern humans, a group of closely related haplotypes at MCPH1, called haplogroup D, and found that it arose from a single copy $\sim 37,000$ years ago and subsequently swept to a very high frequency. These changes probably resulted from the effects of recent population expansion for modern humans and also selection in the lineage leading to H. sapiens (Wang and Su 2004, Evans et al. 2005, 2006). They suggest that this haplogroup originated in a hominin lineage that had diverged from modern humans around 1.1 mya and then introgressed into humans by $\sim 37,000$ years ago. If true, then this finding would imply that admixture occurred between archaic and modern humans (Evans et al. 2005, 2006).

ASPM has also undergone strong positive selection over the course of human and primate evolution. Its sequence shows evidence of accelerated evolution in the African hominoid clade, which preceded hominin brain expansion by several million years, as well as during recent human evolution (Evans et al. 2004b, Kouprina et al. 2004). In fact, it has been estimated that, on average, ASPM fixed one advantageous amino acid change in every 300,000-400,000 years since the human lineage diverged from chimpanzees some 5-6 mya (Evans et al. 2004b, Kouprina et al. 2004). Furthermore, the regions of the ASPM gene under positive selection in primates are also the most highly diverged regions between primates and non-primate mammals (Kouprina et al. 2004). Therefore, current data indicate that the ASPM gene has undergone adaptive evolution in the hominin lineage. Moreover, these findings indicate that brain size seems to be controlled in part through the 
modulation of mitotic spindle activity in neuronal progenitor cells (Bond et al. 2002).

A third gene that may be of some importance for human brain evolution is the Abelson Helper Integration Site 1 (AHI1) locus. This gene is essential for axon path finding from the cortex to the spinal cord and, thus, for normal coordination and gait (Hill and Walsh 2005). It, too, causes severe neurological disease when mutated, including mental retardation and sometimes autism-like symptoms, such as anti-social behavior and perhaps even schizophrenia (Ingason et al. 2007, Alvarez Retuerto et al. 2008). This gene also shows evidence for positive selection in the lineage leading to humans (Ferland et al. 2004). Together, these observations have been interpreted as suggesting that sequence differences in AHI1 may relate not only to human patterns of gait, but also to species-specific social behavior (Ferland et al. 2004, Hill and Walsh 2005).

\section{Brain Systems for Language}

When it comes to modeling brain evolution in apes and humans, relative size does seem to matter. There is a general relationship between brain size and the complexity of hominin behavior as viewed through the lithic production, faunal analysis, coordination of group activities, language ability, and adaptability to numerous different environments. However, more recent studies of brain anatomy employing modern imaging techniques are showing that expanding brain size is only part of the story behind the emergence of modern human cognitive function and behavioral capacities.

Various reports have shown that there are different systems for language in the left hemisphere in the brain (Schoenemann 2006). These include word- and sentence-implementation structures and mediation structures for lexical items and grammar (Fig. 2.13). In addition, the sets of neural structures that represent the concepts themselves are distributed across both right and left hemispheres in many sensory and motor regions. In addition, researchers have identified a parallel pathway to the classical arcuate pathway connecting Broca's and Wernicke's areas (Catani, Jones, and ffytche 2005). Interestingly, this parallel pathway, called Gerschwind's territory, may be important for the acquisition of language in childhood. It is the last area in the brain to mature, and the completion of its maturation generally coincides with the development of reading and writing skills in young children (Catani, Jones, and Ffytch 2005). 


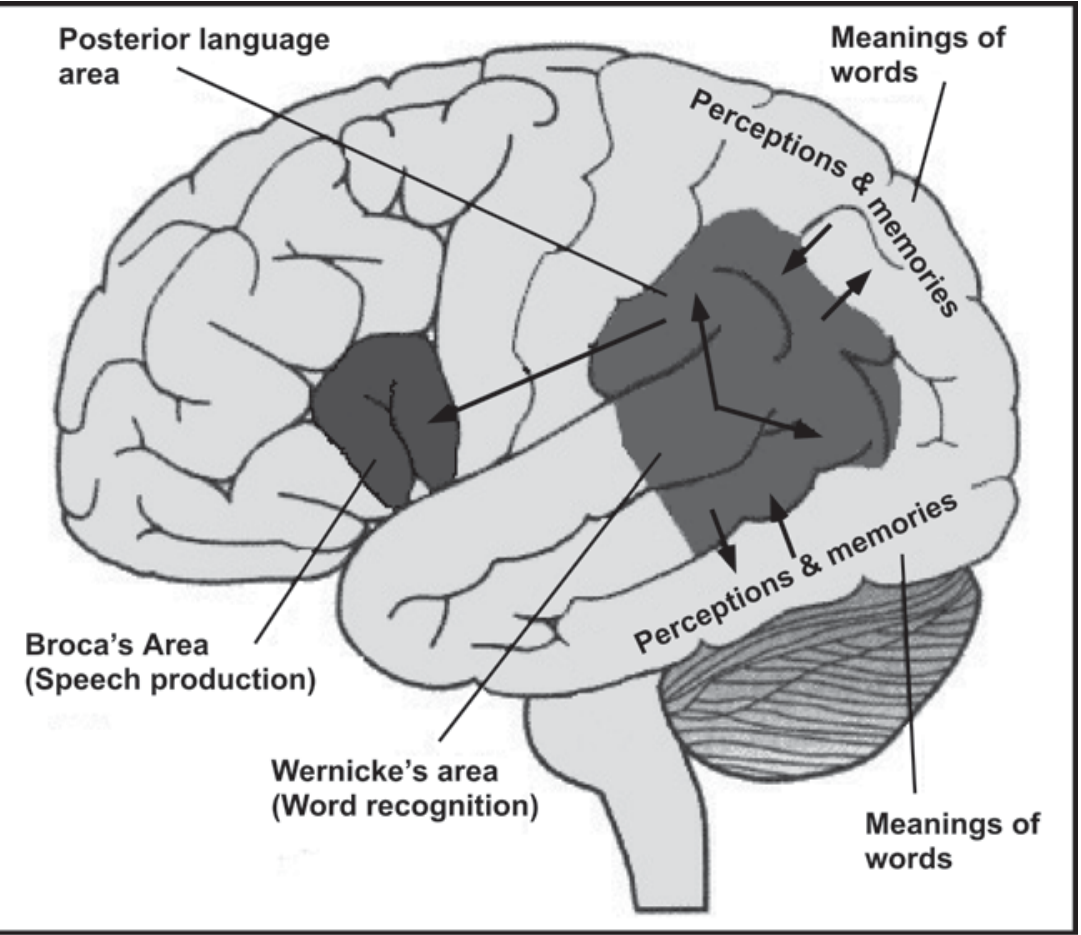

2.13. Language areas of the brain. (Modified after image from http://emedia. leeward.hawaii.edu/hurley/Ling102web/mod5_Llearning/5mod5.2_disorders. htm.)

The identification of different parts of the brain that are involved in language production and comprehension, as well as the evolution of the different parts of the brain in humans and other primates, has been made possible by the use of modern imaging technologies, such as computer tomography (CT) and magnetic resonance imaging (MRI). Studies employing these methods have greatly facilitated cross-species comparisons of brain size, and the examination of developmental features of brain function.

In one such study, Gogtay et al. (2004) investigated the developmental features of brain maturation in apes and humans. Their primary goal was to better understand the anatomical sequence of human cortical gray matter development between the ages of 4 and 21 years. MRI scans showed that higher-order association cortices matured only after the development of the lower-order somatosensory and visual cortices, the functions of which they 
integrate. In addition, the phylogenetically older areas of the brain matured earlier than the newer ones.

In a similar study, Schoenemann, Sheehan, and Glotzer (2005) investigated the extent to which different parts of the brain increased in hominins compared to apes. They focused on the prefrontal cortex, which mediates evolutionarily important behaviors such as planning complex cognitive behaviors, personality expression, decision making, and controlling social behavior, while also generally orchestrating thoughts and actions in accordance with internal goals (Miller and Cohen 2001, Krawczyk 2002, Lebedev et al. 2004). Through the MRI imaging of 11 primates species, Schoenemann, Sheehan, and Glotzer (2005) were able to measure gray, white, and total volumes for both prefrontal and the entire cerebrum for each taxa. They found that the prefrontal white matter showed the largest difference between apes and humans, while gray matter showed no significant difference at all. Schoenemann, Sheehan, and Glotzer (2005) interpreted these results as suggesting that connectional elaboration, as indicated by white matter volume, played a key role in human brain evolution.

Although these studies show that relative brain size, the nature and extent of neuron connections, and the comparative volumes of white and gray matter are of great evolutionary and functional importance in apes and humans, they did not specifically examine the changes in sizes of different parts of the brain through time and across species. In an effort to understand these aspects of hominoid evolution, Rilling (2006) analyzed brain structure sizes across the primate order to determine whether human, ape, and other anthropoid brains are allometrically scaled versions of the same generalized design. His MRI results showed that both human and ape brains exhibited specializations with respect to other anthropoid brains. More specifically,

[a]pe specializations include elaboration of the cerebellum (all apes) and frontal lobes (great apes only), and probably connectivity between them. Human brain specializations include an overall larger proportion of neocortex, with disproportionate enlargement of prefrontal and temporal association cortices, an apparent increase in cerebellar connections with cerebral cortical association areas involved in cognition, and a probable augmentation of intracortical connectivity in prefrontal cortex. (Rilling 2006:65) 
These quantitative and qualitative differences in the brain are undoubtedly linked to the species' cognitive abilities, while the dramatic expansion of higher-order association cortices in humans would have supported the emergence of language abilities in the hominin lineage (Rilling 2006).

Moving from the structural to the cellular level, recent neuroanatomical studies have also yielded insights into the expansion of the brain in apes and humans, and, in particular, developmental aspects of neuron formation. For example, it has been noted that the enlarged cortex of hominoids reflects a longer period of neuronal formation during prenatal development, meaning that each dividing progenitor cell undergoes more cell cycles before stopping cell division (Kornack and Rakic 1998). In addition, the longer period of neurogenesis adds novel neurons to the cortex, thereby allowing the cortical circuit diagram to develop differently in primates compared to other mammals. Upper cortical layers, generated late in neurogenesis, are overrepresented in the primate cerebral cortex, especially in humans (Marin-Padilla 1992). Additionally, special cell types, such as spindle cells (specialized, deeplayer neurons), are unique to primates (Allman, Hakeem, and Watson 2002). Thus, the primate brain is noteworthy not only for its size and complexity relative to other mammalian species, but also for the nature and extent of neuronal connections within the brain itself (Hill and Walsh 2005).

\section{Myosin Gene Evolution}

While most studies of the emergence of language production and comprehension have focused on genes influencing brain anatomy or the analysis of cognitive and neurological phenomena underlying its function, other researchers have taken a different approach to understanding the evolution of large brains in hominins. In one such study, Stedman et al. (2004) examined the masticatory muscles in humans and several primates, as well as one of the genes involved in producing them. They noted that powerful masticatory muscles are found in most primates, including chimpanzees and gorillas, and were a prominent part of the adaptive strategies of Australopithecus and Paranthropus. In contrast, these muscles are considerably smaller in both modern and fossil members of the genus Homo. The evolving hominin masticatory apparatus, which can be traced back to a Late Miocene, chimpanzee-like morphology (White et al. 2000), shifted toward a pattern of gracilization nearly simultaneously with accelerated encephalization in early Homo (Tobias 1991, Schoenemann 2006). 


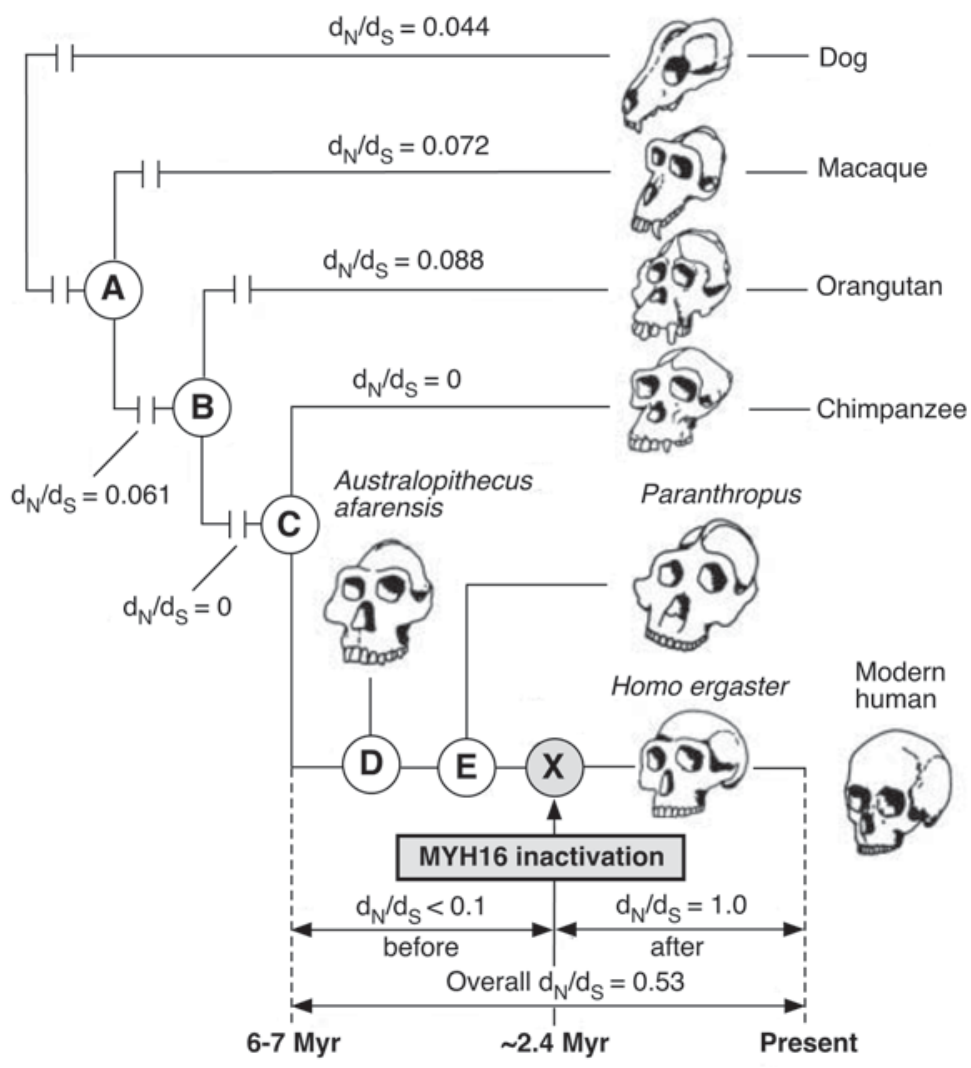

2.14. Divergence of the myosin heavy chain 16 (MYH16) gene. The data are based on nucleotide sequences for the six largest exons in the rod-coding domain. Individual mutations are indicated by $N$ for non-synonymous or $S$ for synonymous. $d_{N}$ and $d_{S}$ refer to the mutational rates normalized to the number of relevant sites $(840 \mathrm{~N}, 225 \mathrm{~S})$ in pairwise comparisons between MYH16 orthologues of extant species and inferred ancestors at nodes $A, B$, and $C$. The atypically high $d_{N} / d_{S}$ in the human ancestral lineage (significant, $P=0.0527$ ) indicates a loss of negative Darwinian selection approximately 2.4 million years ago (Myr). (Reprinted by permission from Macmillan Publishers Ltd: Nature 428 [2004], H.H. Stedman et al., pp. 415-18, Fig. 4.)

In support of this view, Stedman et al. (2004) found that the gene encoding the predominant myosin heavy chain (MYH) expressed in these muscles was inactivated by a frameshift mutation after the lineages leading to humans and chimpanzees diverged. Based on histological comparisons, they noted that the mutation was associated with marked size reductions in in- 
dividual muscle fibers and entire masticatory muscles in modern humans compared to great apes (Fig. 2.14). Coalescence estimates using primate and human MYH sequences indicated that the frameshift mutation appeared approximately 2.4 mya. This date predates the appearance of modern human body size (Walker and Leakey 1993, Pearson 2000, Aiello and Wells 2002, Antón and Swisher 2004, Ruff 2008) and the emigration of Homo from Africa (Vekua et al. 2002, Antón and Swisher 2004). Given these findings, Stedman et al. (2004) asserted that the reduction in the mass of the temporalis muscle allowed the cranium/brain to expand in size. Thus, in their view, the MYH mutation facilitated greater encephalization in the hominin lineage due to removing physiological and structural constraints on brain and cranial growth.

It should be noted here that the proposed date for the MYH mutation coincides with the timing of several key transitions in hominin biology and behavior. The first of these is the beginning of the Oldowan lithic tradition in east Africa, which is generally viewed as being initiated by early Homo (Haile-Selassie 2001, Panger et al. 2002). The use of tools is viewed as being significant in that it demonstrates the existence of mental templates in the minds of early hominins, not to mention the more systematic use of raw materials, the processing of food, and task specialization.

\section{Sexual Selection}

Yet another factor that may be relevant to understanding the process of increased brain size in humans is sexual selection. Schillaci (2006) examined the relationship between brain size and sexual selection in terms of sperm competition and male competition for mates. While observing no significant relationship between relative brain size and sperm competition as measured by testis size in primates, he did find a significant negative relationship between relative brain size and the level of male competition for mates. These results suggested to him that the largest relative brain sizes among primate species are associated with monogamous mating systems, suggesting that primate monogamy may require greater social acuity and abilities of deception.

\section{SUMMARY}

This chapter outlines the major evolutionary events in modern human evolution, including the transitions between different hominid and hominin forms. We have also evaluated the emergence of human characteristics in 
the context of broader primate and mammalian evolutionary patterns. The totality of the evidence shows that, while having roots in primate and hominin prehistory, modern humans have undergone significant biological and behavioral changes over the last 2 million years.

Overall, genomic comparisons clearly show that the human genome has undergone profound changes in terms of its size, content, and regulation relative to those of chimps, gorillas, and other primate species. While these interspecific comparisons have identified a number of genes that have undergone selection, or relaxed selection, in the hominin lineage, ongoing analysis may well identify additional loci of similar evolutionary importance. New sequence data will also help to improve estimates of the neutral substitution rate, i.e., the rate at which fixed differences between species accumulate under neutral evolution, hence, the timing of the divergence of ape and human lineages (e.g., Sabeti et al. 2006). Furthermore, we need to improve our understanding of the likely functional outcomes of these genetic changes, such as the effects of specific mutations on protein structure and function, and the way that noncoding regulatory regions of the genome work.

While there is close genetic affinity betweem apes and humans, such that these taxa share over $96 \%$ of their genomic sequences, it is also increasingly evident that there are significant differences in the expression and regulation of these genes in the two species, as well as specific allelic and chromosomal changes (e.g., segmental duplications) that led to the phenotypic, physiological, and behavioral differences seen in them. Each lineage has also been affected by selection in different ways, and, for humans, the extent to which cultural and sexual selection have shaped genetic variation requires fuller resolution.

Current evidence gives us a better sense of the basis of cognitive differences between apes and humans, and the evolutionary forces that have influenced them. While relative brain size is clearly one feature that distinguishes humans from apes, it is only one dimension of the transformation of the hominid brain. In fact, we observe the expansion of different areas of the brain in humans relative to other primates, including the cortical areas related to higher cognitive function, as well as differences in neuronal density and neuroanatomical architecture. Although it is possible to infer when some of the changes occurred in the hominin lineage, e.g., the emergence of the genus Homo in the Pliocene-Pleistocene boundary, the timing 
of most of these transitions remains speculative. However, it is clear that the hominin brain has been transformed in a manner that now allows us to have articulate speech, abstract reasoning, and complex cultural behavior in contrast to other hominoid apes.

The conditions under which the transformation of the genus Homo took place - which shaped the evolution of the species or lineage-are still incompletely understood. Major climate changes may have triggered a shift in subsistence towards broader spectrum diet and greater consumption of meat, and accompanying behavior changes (hunting/scavenging). This dietary shift led to the transformation in gut morphology of hominids, setting the biological foundation for the later encephalization of the lineage, and perhaps also the bioenergetic basis for more hominin-like forms of bipedalism, which permitted the expansion of the lineage outside of its Africa homeland.

Even though these findings have helped us reconstruct the emergence and evolution of the hominin lineage, there are still many questions and issues concerning this process that remain to be resolved. Among these are the timing of emergence of modern human language, the selective forces that promoted rapid brain size growth, and the relationship between brain size and complexity and primate and hominin linguistic and cultural abilities. Ongoing and future work, particularly research at the genomic level, should provide new insights into these questions and tell us more about the evolution of the modern human mind, brain, and culture.

\section{NOTES}

2.1 Hominins are humans or human ancestors. They include all of the Homo species (Homo sapiens, H. ergaster, H. rudolfensis), all of the australopithecines (Australopithicus africanus, A. boisei, etc.), and other ancient forms like Paranthropus and Ardipithecus.

2.2 A clade is a group of species whose members share homologous features derived from a common ancestor.

2.3 Gracile and robust australopithecines are differentiated by the size of their jaws, teeth and cranial features, among other traits.

2.4 Sexual dimorphism refers to the difference in size between males and females of a species; males are usually slightly to considerably larger than females in hominid and hominin species.

2.5 Sympatry refers to species whose ranges overlap or are even identical such that they occur together, at least in some places. If they are closely related, they may have arisen through sympatric speciation. 
2.6 Ontogeny is the origin and development of an individual organism from embryo to adult.

2.7 Haploinsufficiency is the situation where the total amount of a gene product (protein) produced by cells is half of the normal amount, thereby causing the cell to function abnormally. It usually occurs when one of two functional copies of a gene is inactivated by mutation, with the resulting reduction of gene function producing an abnormal phenotype. 
This page intentionally left blank 